\title{
ORIGINAL ARTICLE SARA and RNF11 interact with each other and ESCRT-0 core proteins and regulate degradative EGFR trafficking
}

\author{
E Kostaras ${ }^{1,2}$, G Sflomos ${ }^{1,2}$, NM Pedersen ${ }^{3}$, H Stenmark ${ }^{3}$, T Fotsis ${ }^{1,2,4}$ and C Murphy ${ }^{2,4}$
}

\begin{abstract}
Smad anchor for receptor activation (SARA) is highly enriched on endocytic membranes via binding to phosphatidylinositol 3-phosphates through its FYVE (Fab1p-YOTB-Vps27p-EEA1) domain. SARA was originally identified as a protein that recruits non-phosphorylated SMAD2/3 to the activated TGF $\beta$ receptors for phosphorylation, but later reports suggested a regulatory role in endocytic trafficking. Here we demonstrate that the ubiquitin ligase RNF11 is a SARA-interacting protein residing on early and late endosomes, as well as the fast recycling compartment. RNF11 and SARA interact with the ESCRT-0 subunits STAM2 and Eps15b, but only RNF11 associates with the core subunit Hrs. Both gain- and loss-of-function perturbation of RNF11 and SARA levels result in delayed degradation of epidermal growth factor (EGF)-activated EGF receptor (EGFR), while loss-of-function sustained/enhanced EGF-induced ERK1/2 phosphorylation. These findings suggest that RNF11 and SARA are functional components of the ESCRT-0 complexes. Moreover, SARA interacts with clathrin, the ESCRT-I subunit Tsg101 and ubiquitinated cargo exhibiting all the properties of Hrs concerning ESCRT-0 function, indicating that it could substitute Hrs in some ESCRT-0 complexes. These results suggest that RNF11 and SARA participate structurally and functionally in the ESCRT-dependent lysosomal degradation of receptors. As a consequence, the negative influence that perturbation of RNF11 and SARA levels exerts on the lysosomal degradation of EGFRs could underscore the reported overexpression of RNF11 in several cancers. In these cancers, deficient termination of the oncogenic signaling of mutated receptors, such as the EGFRs, through suboptimal lysosomal degradation could contribute to the process of malignant transformation.
\end{abstract}

Oncogene (2013) 32, 5220-5232; doi:10.1038/onc.2012.554; published online 10 December 2012

Keywords: ESCRT-0; membrane trafficking; RNF11; SARA

\section{INTRODUCTION}

Signaling receptors undergo internalization via various endocytic entry routes, ${ }^{1}$ clathrin-mediated endocytosis being the most known. Cargo, entering via these alternative routes, is transported to a series of intracellular compartments from where it is either recycled to the cell surface via recycling endosomes or directed to degradative compartments (late endosomes and lysosomes). Thus, the trafficking endomembrane system determines the strength and duration of signaling responses by controlling sorting events such as recycling and transport to the lysosomal compartment for ligand/receptor degradation, but also by recruiting downstream effectors of signaling complexes.

FYVE (Fab1p-YOTB-Vps27p-EEA1) domain-containing proteins bind to phosphatidy linositol 3-phosphate, which is highly enriched in endocytic membranes. As a consequence, FYVE domain-containing proteins are recruited to these compartments serving as regulators of endocytic membrane trafficking and signaling, albeit the latter function is less common. Early endosomal antigen (EEA1), hepatocyte growth factor-regulated tyrosine kinase substrate (Hrs) and Smad anchor for receptor activation (SARA) are three members of this family. SARA was originally identified as a protein that functions to recruit nonphosphorylated SMAD2/3 to the activated TGF $\beta$ receptors for phosphorylation. Indeed, SARA binds SMAD2/3 proteins via a specific domain called SMAD-binding domain. ${ }^{2,3}$ Moreover, SARA binds and recruits protein phosphatase 1 to a specific domain downstream of SMAD-binding domain, thereby mediating TGF $\beta$ receptor dephosphorylation. ${ }^{4}$

In addition to the above-mentioned signaling properties, it has been reported that SARA also regulates trafficking. ${ }^{5}$ Indeed, overexpression of SARA caused enlargement of early endosomes in a Rab5-dependent manner. Moreover, in SARA overexpressing cells, the number of transferrin ( $T f$ ) receptors on the cell surface was reduced as was Tf recycling, suggesting that Tf and Tf receptor remained associated in the expanded early endosomes. ${ }^{5}$ Furthermore, SARA has been shown to regulate the vesicular targeting of rhodopsin-bearing axonemal vesicles in the lightsensing organelle in mammalian rods. $^{6}$ Because SARA appears to regulate both signaling and trafficking in the endocytic compartment, we have undertaken the task of identifying SARAinteracting proteins aimed at understanding in more depth the role of SARA in signaling and trafficking and the links thereof.

\section{RESULTS}

RNF11 is a SARA-interacting protein

To identify SARA-interacting proteins, a yeast two-hybrid $(\mathrm{Y} 2 \mathrm{H})$ screen was performed using a human placental cDNA library as prey and the carboxy-terminal region of SARA (SARA $\triangle 1-664$ ) as bait. Fifteen $\mathrm{Y} 2 \mathrm{H}$ clones identified RNF11 RING-H2 E3 ligase (62-154 amino acid (aa)) as a SARA-interacting protein.

\footnotetext{
${ }^{1}$ Laboratory of Biological Chemistry, Medical School, University of loannina, loannina, Greece; ${ }^{2}$ Department of Biomedical Research, Foundation for Research and TechnologyHellas, Institute of Molecular Biology and Biotechnology, University Campus of Ioannina, loannina, Greece and ${ }^{3}$ Centre for Cancer Biomedicine, Faculty of Medicine, University of Oslo, Oslo, Norway. Correspondence: Dr C Murphy, Department of Biomedical Research, Foundation for Research and Technology-Hellas, Institute of Molecular Biology and Biotechnology, University Campus of Ioannina, 45110 loannina, Greece.

E-mail: cmurphy@cc.uoi.gr

${ }^{4}$ These authors equally contributed to this work.

Received 10 April 2012; revised 28 September 2012; accepted 4 October 2012; published online 10 December 2012
} 
a

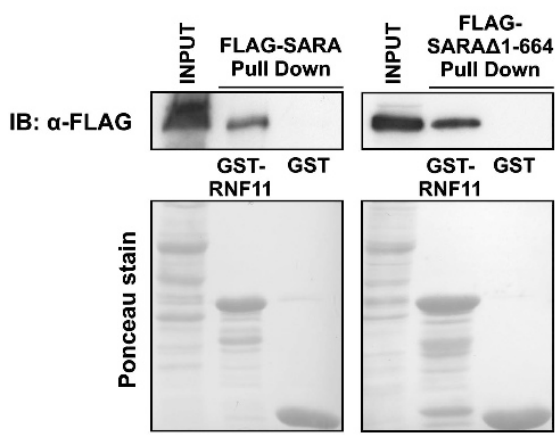

b

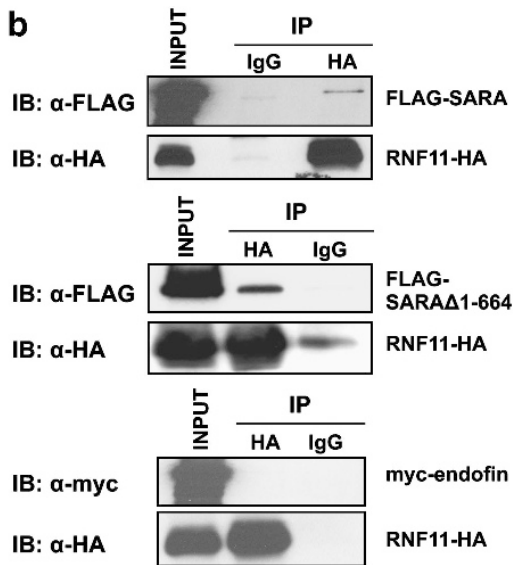

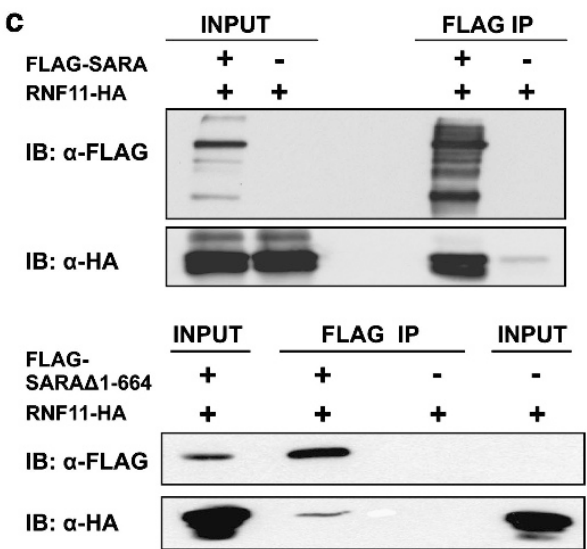

Figure 1. SARA and RNF11 interact in vitro and in vivo: (a) GST pull-down assay: HEK293 cell lysates expressing either FLAG-SARA or FLAGSARA $\triangle 1-664$ were incubated with GST-RNF11 or GST alone. The complexes were immunoblotted with an $\alpha$-FLAG antibody. The lower panel in each case shows a ponceau stain of the GST proteins. (b, c) Co-immunoprecipitation of overexpressed proteins: (b) BHK (upper and lower panels) or HEK293 (middle panel) cells overexpressing RNF11-HA and FLAG-SARA, FLAG-SARA $\Delta 1-664$ or myc-endofin were lysed using 0.5\% NP-40, and $\alpha-\mathrm{HA}$ or rat IgG antibody immunoprecipitations were performed. Immunocomplexes were washed, analyzed by SDS-PAGE and immunoblotted with an $\alpha$-HA antibody to confirm RNF11-HA immunoprecipitation and with $\alpha$-FLAG or $\alpha$-myc antibody to detect the coimmunoprecipitation of FLAG-SARA, FLAG-SARA $1-664$ or myc-endofin. (c) BHK cells (upper) or HEK293 cells (lower) were transfected with FLAG-SARA and RNF11-HA or infected with an adenovirus expressing FLAG-SARA $\Delta 1-664$ and transfected with RNF11-HA, respectively. FLAG proteins were immunoprecipitated using $\alpha$-FLAG-affinity conjugated agarose beads.

Indeed, glutathione S-transferase (GST)-RNF11 pulled down both FLAG-SARA and FLAG-SARA $\triangle 1-664$ (Figure 1a). Moreover, FLAG-SARA or FLAG-SARA $\triangle 1-664$ was detected in the immunoprecipitates of RNF11-HA (Figure 1b), whereas myc-endofin, a protein with close homology to SARA, was not (Figure 1b). In agreement, RNF11-HA was detected in FLAG-SARA and FLAGSARA $\triangle 1-664$ immunoprecipitates (Figure 1c). Though the interaction of the endogenous proteins was not feasible because of lack of specific antibodies against RNF11, these experiments clearly show that RNF11 is a SARA-interacting protein.

RNF11 localizes to the early and late endocytic, as well as the fast recycling compartments, in a SARA-independent manner

RNF11 colocalized with EEA1 (33 $\pm 5.7 \%)$ (Figure $2 \mathrm{~A}$, a), Rab5 $(46 \pm 8 \%)$ (Figure $2 \mathrm{~A}, \mathrm{~b})$ and Rabenosyn-5 (34 $\pm 7.5 \%$ ) (Figure $2 \mathrm{~A}, \mathrm{C}$ ), all known markers of early endosomes. ${ }^{7-9}$ Moreover, RNF11 colocalized extensively $(42 \pm 8 \%$ ) with Rab7 (Figure $2 A, d$ ), a late endocytic marker, ${ }^{10}$ whereas colocalization with the lysosomal markers Lamp1 (Figure 2A, e) and CD63 (Figure 2B, f) was minimal (approximately 12\%). Moreover, the RNF11 vesicles positive for Rab7 are mostly Lamp1 and CD63-negative, suggesting that RNF11 colocalizes minimally with the lysosomal compartment (Supplementary Figure 3B, compare arrowheads and quantitation of triple colocalization). RNF11 did not exhibit any colocalization with Rab11 (Figure 2B, g and Supplementary Figure 3A), a marker of the perinuclear recycling compartment, but exhibited extensive colocalization with Rab4 (Figure 2B, h and Supplementary Figure $3 \mathrm{~A}$ ) and Tf (Figure 2B, i), markers of the fast recycling compartment and the fast recycling and perinuclear recycling compartment, respectively. Early endosomes in the cell periphery are mostly Rab5- and Rab4-positive and rarely contain Rab11, and as one moves to the center of the cell, the endosomes are mostly Rab4 and Rab11 positive. ${ }^{11}$ It seems that RNF11 localizes to the peripheral Rab5/Rab4-positive compartment. To rule out perturbation of the endocytic compartment by transiently overexpressing Rab proteins, we further investigated RNF11 localization in A431 cell lines stably expressing either Rab11 or Rab4, almost at endogenous levels. ${ }^{11}$ The results are comparable to those observed in experiments with transient transfection of human umbilical vein endothelial cells. RNF11 colocalizes extensively with Rab4a, but not with Rab11 (Supplementary Figure 3A). Finally, RNF11 did not colocalize with Caveolin-1 (Figure 2B, j), a caveolar marker. ${ }^{12,13}$ Furthermore, green fluorescent protein (GFP)-tagged (Figure $3 \mathrm{Aa}-\mathrm{c}$ ) and endogenous SARA (Figure $3 A d-f$ ) colocalized extensively ( $42 \pm 5$ and $47 \pm 12 \%$, respectively) with RNF11-mcherry in human umbilical vein endothelial cells. Moreover, EEA1, RNF11-mcherry and GFP-SARA, 
all colocalized on the same vesicles (early endosomes) (Figure 3B). Untagged RNF11 colocalized extensively with EEA1 on the endocytic compartment, excluding any influence of the tag on the localization (Figure 3C). Finally, RNF11 was clearly associated with vesicular structures (Figure 3Da-C) positive for EEA1 (data not shown), despite silencing of ZFYVE9 (SARA) gene (Figure 3E). In summary, RNF11 is localized to the early and late endocytic, as well as the fast recycling compartments. However, its interaction with SARA is not responsible for the endocytic recruitment of RNF11.

$\mathrm{N}$-myristoylation and rapid endocytosis contribute to the accumulation of RNF11 in early endosomes

RNF11 has several known domains, such as the PY motif (aa 3740), the RING-H2 domain (aa 99-139) ${ }^{14}$ and a myristoylation sequence at the $\mathrm{N}$-terminus that may explain its endosomal localization. RNF11 mutants either in the PY motif or the RING-H2 domain (RNF11-HA Y40A and RNF11-HA C99A, respectively) exhibited a vesicular (punctate) staining, a property shared with RNF11 tagged at the C-terminus (RNF11-HA) (Figures 4Aa, $\mathrm{d}$ and e). On the contrary, N-terminal-tagged RNF11 (HA-RNF11) and a myristoylation-preventing RNF11 mutant (RNF11-HA G2A) localized to the cytosol and nucleus (Figure $4 A b$ and $c$ ), suggesting that $\mathrm{N}$-myristoylation is a prerequisite for the endosomal localization of RNF11.

Because we did not detect RNF11 at the plasma membrane, despite the membrane anchoring myristoylation, we assumed that RNF11 is rapidly trafficked to early and late endosomes. Indeed, blocking internalization by overexpressing a dominant-negative form of Dynamin I (K44A) trapped RNF11-mcherry at the cell
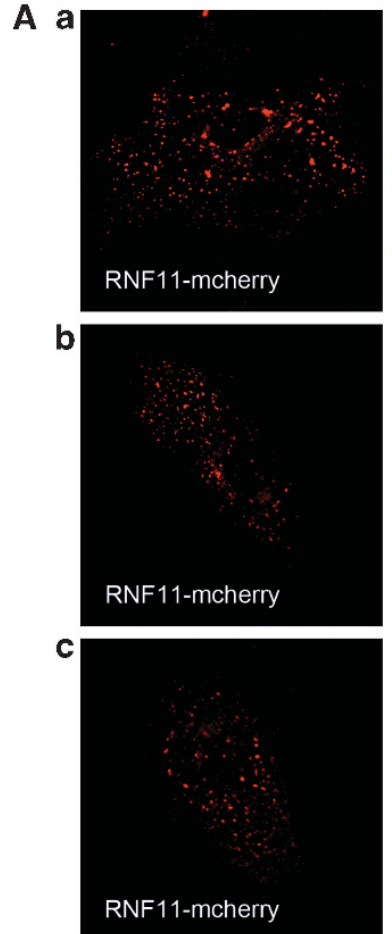

d
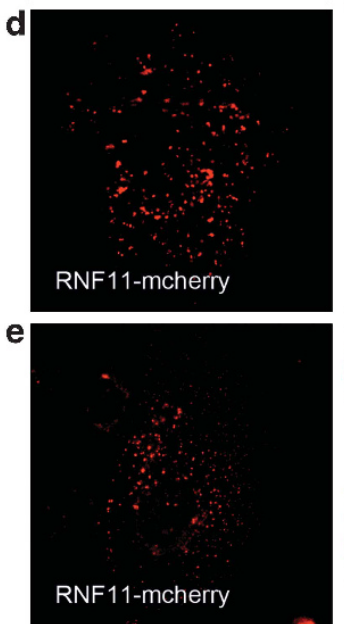
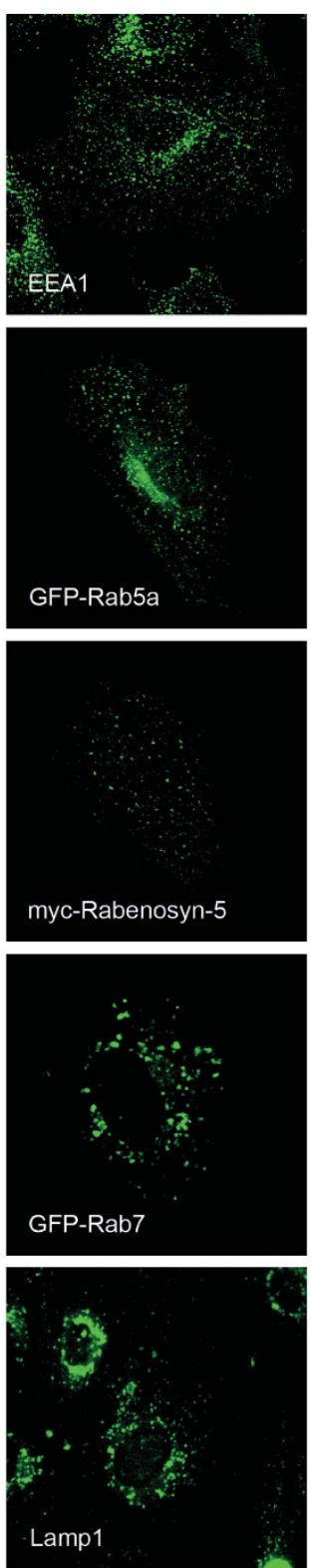
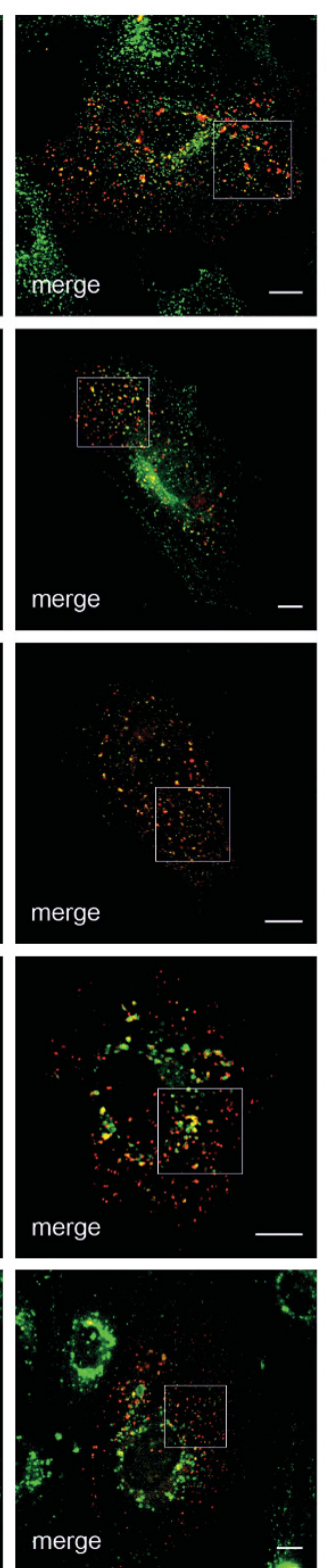
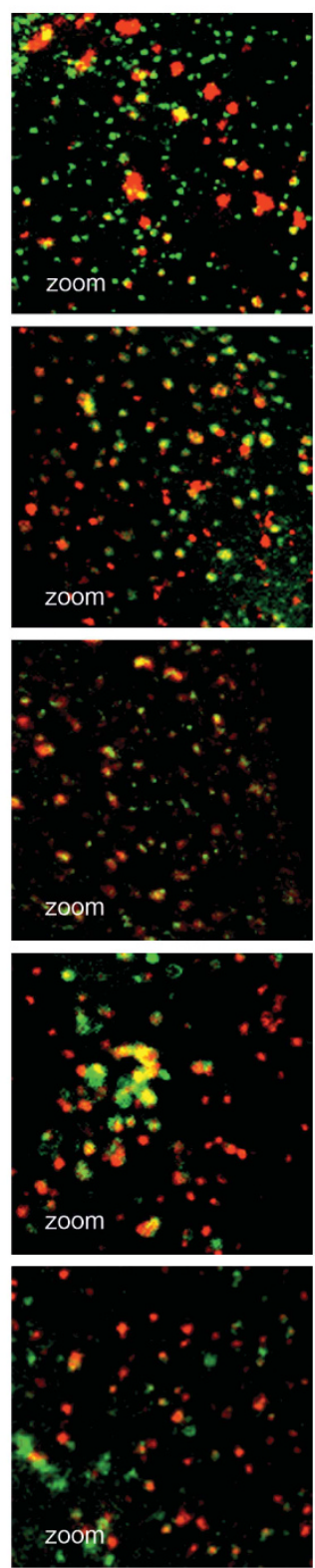

Figure 2. RNF11 localizes to the early, late and fast recycling endocytic compartments: (A, B) human umbilical vein endothelial (HUVE) cells were transiently transfected with RNF11-mcherry alone (a, e) or together with GFP-Rab5a (b), myc-Rabenosyn-5 (c), GFP-Rab7 (d), GFP-Rab11 (g), GFP-Rab4a (h) and GFP-Caveolin-1 (j). In the case of EEA1 (a), Lamp1 (e) and CD63 (f), $\alpha$-EEA1, $\alpha$-Lamp1 and $\alpha$-CD63 antibodies were used, whereas myc-Rabenosyn-5 (c) was visualized using the 9E10 antibody. In panels (f) and (i), HUVE cells were infected with RNF11-GFP adenovirus, and in panel (i), tritc-Tf $(50 \mu \mathrm{g} / \mathrm{ml})$ was added at $37^{\circ} \mathrm{C}$ for $30 \mathrm{~min}$. Merged images and zoomed inserts are shown in each case. Scale bars, $10 \mu \mathrm{m}$. 
B

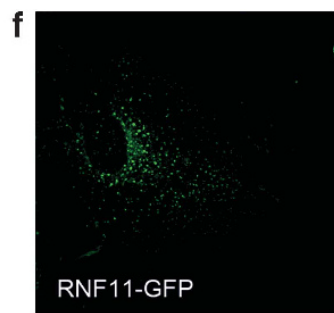

g

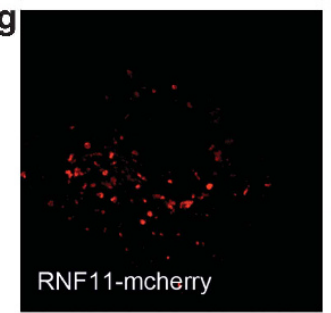

h

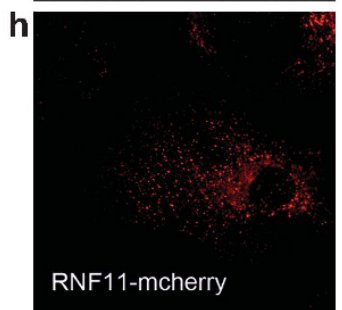

i
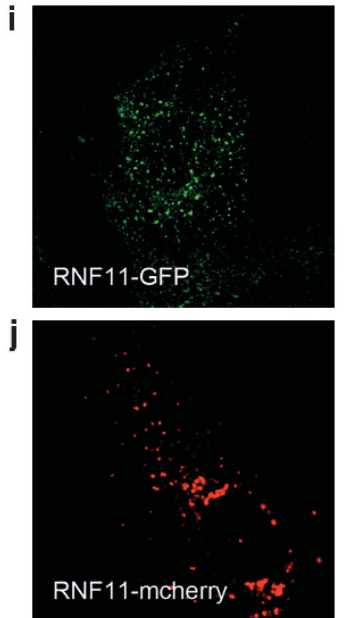
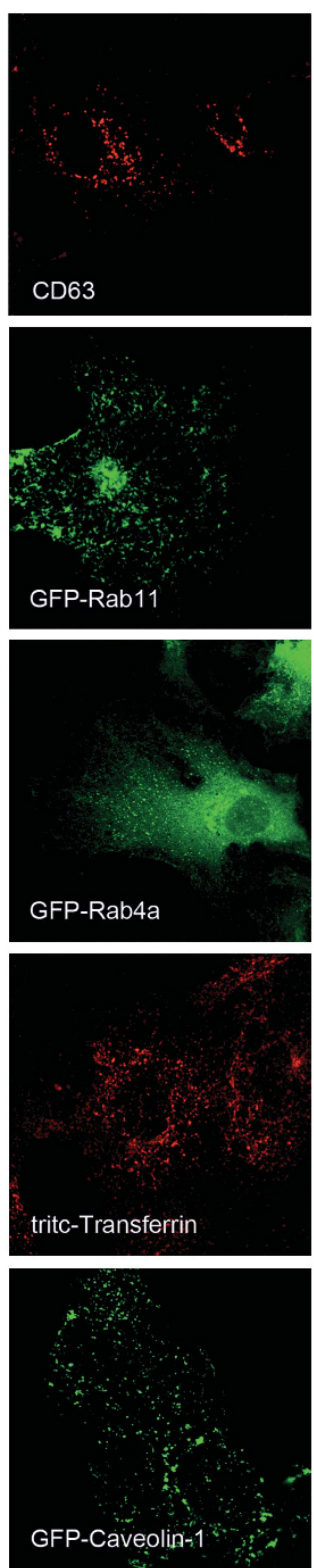
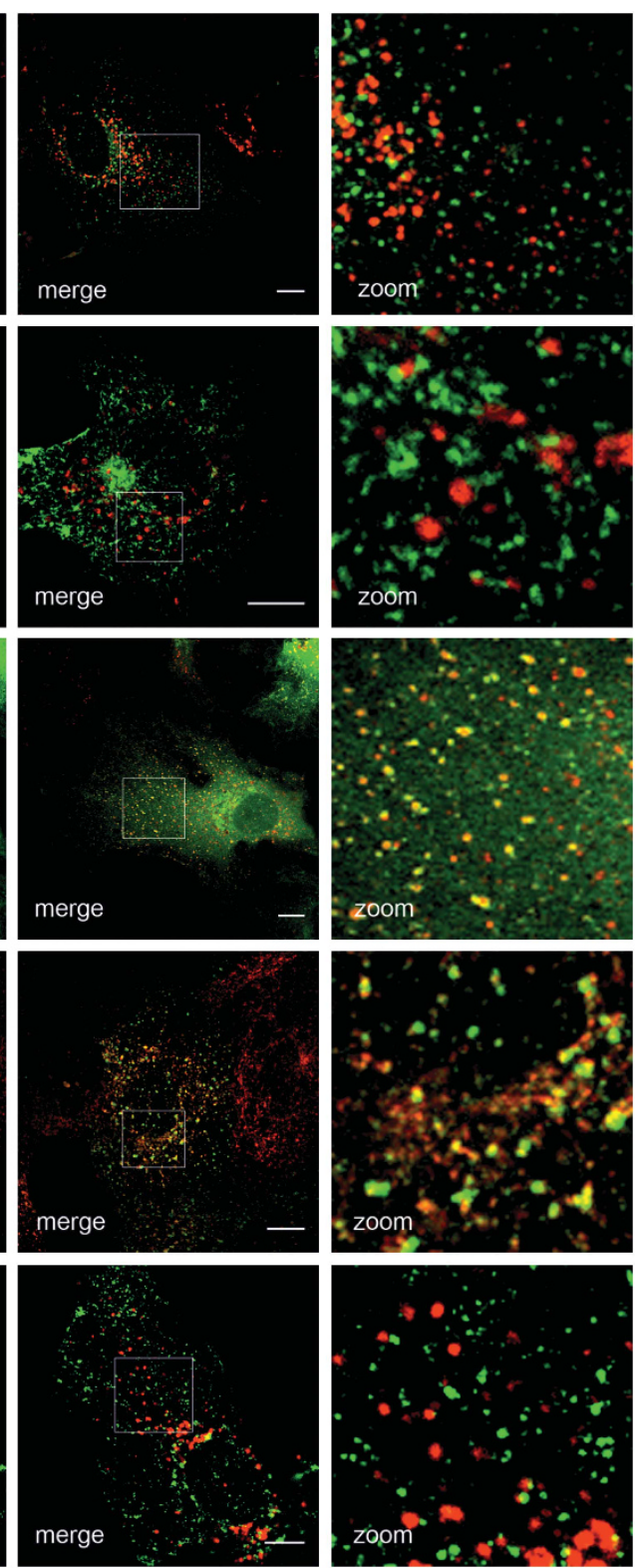

Figure 2. Continued.

surface (Figure 4Ba and $\mathrm{c}$ ). The same was true, when we inhibited endocytosis using two Dynamin II-specific small interfering RNAs (siRNAs) (Figure 4Cb and c). These data indicate that, following synthesis, RNF11 reaches the plasma membrane accumulating in the endocytic compartment via rapid endocytosis.

Mapping the domains responsible for the RNF11-SARA interaction We generated overlapping carboxy-terminal constructs of SARA (Figure $5 \mathrm{a}$ ), covering the $\mathrm{Y} 2 \mathrm{H}$ bait sequence, and tested their interaction with GST-RNF11. SARA-1 (667-926 aa) interacted with GST-RNF11, whereas SARA-2 (906-1204 aa) and SARA-3 (1170-1323 aa) exhibited no interaction (Figure 5b). Likewise, deletion mutants of RNF11 (Figure 5c) indicated that GST-RNF11 and GST-RNF11 $\Delta 1-58$, but not GST-RNF11 $\Delta 59-95$, GSTRNF11 $196-154$ and GST-RNF11 59-95, interacted with SARA $\Delta 1-$ 664 (Figure 5d). These results indicate that both aa 59-95 and RING-H2 domains of RNF11 are required for binding to SARA-1. Indeed, FLAG-SARA $\Delta 1-664$ did not co-immunoprecipitate RNF11-HA $\triangle 59-95$ or RNF11-HA C99A, a mutant of RNF11 in which a cysteine in the RING-H2 domain has been mutated to alanine (Figure 5e).

The RNF11-SARA complex is associated with the ESCRT-0 core proteins

RNF11 binds both homologous to the E6-AP carboxyl terminus (HECT)-type E3 ubiquitin ligases (Nedd4, AIP4, Smurf1 and Smurf2) and deubiquitinating enzymes, such as $\mathrm{AMSH}^{14,15}$ suggesting that it may participate in lysosomal targeting of cargo. Interestingly, STAM2 and Eps15, two subunits of the ESCRT-0 machinery ${ }^{16}$ that participates in the lysosomal trafficking of ubiquitinated cargos, have been suggested to be putative RNF11-interacting proteins in a $\mathrm{Y} 2 \mathrm{H}$ screen. ${ }^{15,17}$ Indeed, GST-RNF11 specifically pulled down myc-STAM2 (Figure 6A, left upper panel), and myc-STAM2 was specifically co-immunoprecipitated by RNF11-HA (Figure 6A, right upper panel). In agreement, overexpressed RNF11-HA and mycSTAM2 colocalize on punctate structures in BHK cells (Figure 6A, lower panel). The same was true for SARA, myc-STAM2 was detected in the $\alpha$-FLAG immunoprecipitates of BHK cells 
A
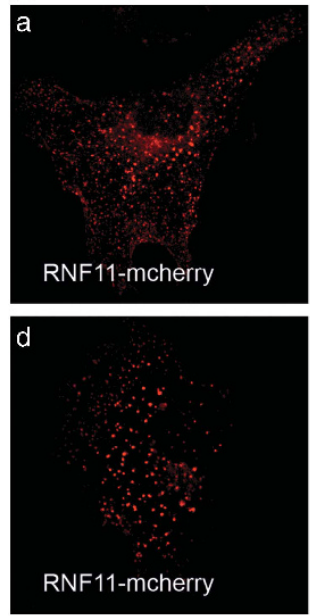

B

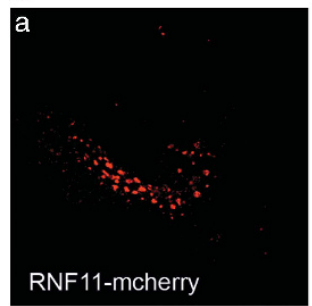

C

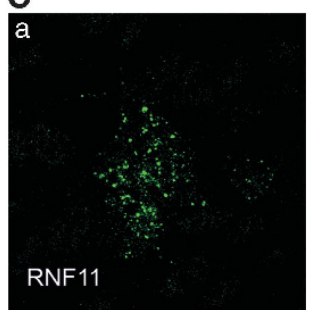

D

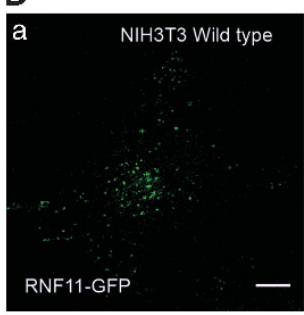

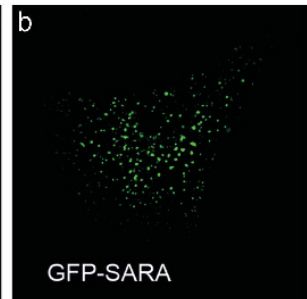
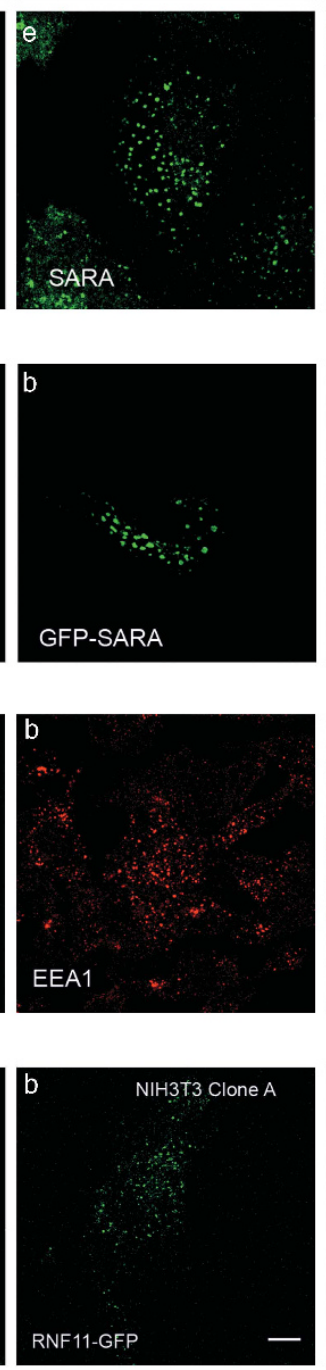
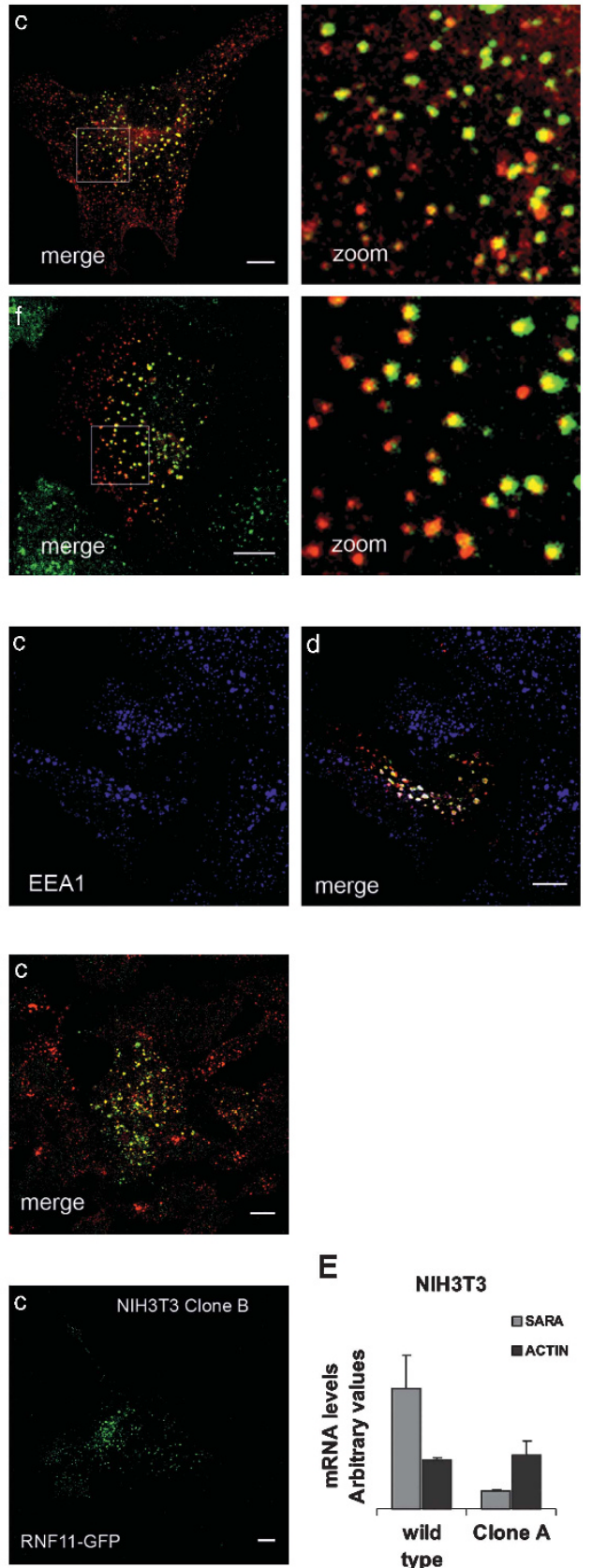

E

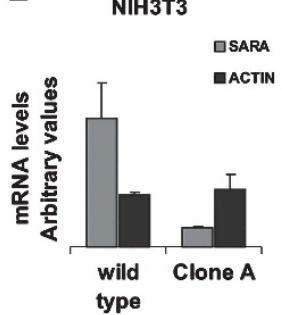

Figure 3. (A) RNF11 colocalizes with overexpressed and endogenous SARA: human umbilical vein endothelial (HUVE) cells were transiently transfected with RNF11-mcherry alone (d-f) or together with GFP-SARA (a-c). Endogenous SARA was detected with rabbit $\alpha$-SARA antibody (e). (B) RNF11 colocalizes with SARA on early endosomes: HUVE cells were transiently co-transfected with RNF11-mcherry (a) and GFP-SARA (b), fixed and processed for indirect immunofluorescence with an $\alpha$-EEA1 antibody (c). (C) Untagged RNF11 colocalize with EEA1: HEK293 cells were transiently transfected with wild-type RNF11, fixed and processed for indirect immunofluorescence with rabbit $\alpha$-RNF11 and mouse $\alpha$-EEA1 antibody. (D) RNF11 localizes on endosomes independently of SARA: Wild-type NIH3T3 cells (a) or stable clones expressing an shRNA of SARA ( $b$ and $c$ ) were transfected with RNF11-GFP, fixed and processed for immunofluorescence. (E) SARA and actin levels were quantitated by qRT-PCR on total RNA isolated from NIH3T3 cells used in Figure 3D. Error bars represent the s.d. of the mean. Scale bars, $10 \mu \mathrm{m}$.

overexpressing myc-STAM2 and FLAG-SARA (Figure 6B). Interestingly, only Eps15b was co-immunoprecipitated by RNF11-HA, but not Eps15 (Figure 6C).

From these experiments, it was evident that RNF11 interacted with SARA, STAM2, and Eps15b and SARA interacted with RNF11 and STAM2. This data indicated that RNF11 and SARA might be in complex with ESCRT-0. Indeed, FLAG-SARA, myc-STAM2 and mycEps15b were all co-immunoprecipitated by RNF11-HA (Figure 6D). On the other hand, Eps15b and STAM2 also exist in complex with Hrs in ESCRT-0 machinery. RNF11-HA interacted with Hrs when both proteins were overexpressed in BHK cells (Figure 6E), but, despite our attempts, SARA and Hrs did not co-immunoprecipitate (Figure 6E).

$\mathrm{Hrs}$ is capable of interacting with clathrin ${ }^{18}$ and the ESCRT-I complex via the TSG101 subunit; ${ }^{19}$ properties that we show here are shared by SARA. Indeed, analysis of the SARA sequence suggested the presence of a potential clathrin box at aa 637-641 (LLYMD) and FLAG-SARA interacted with GST-clathrin (Figure 7a) in pull-down assays. Also, when GFP-SARA was overexpressed in 

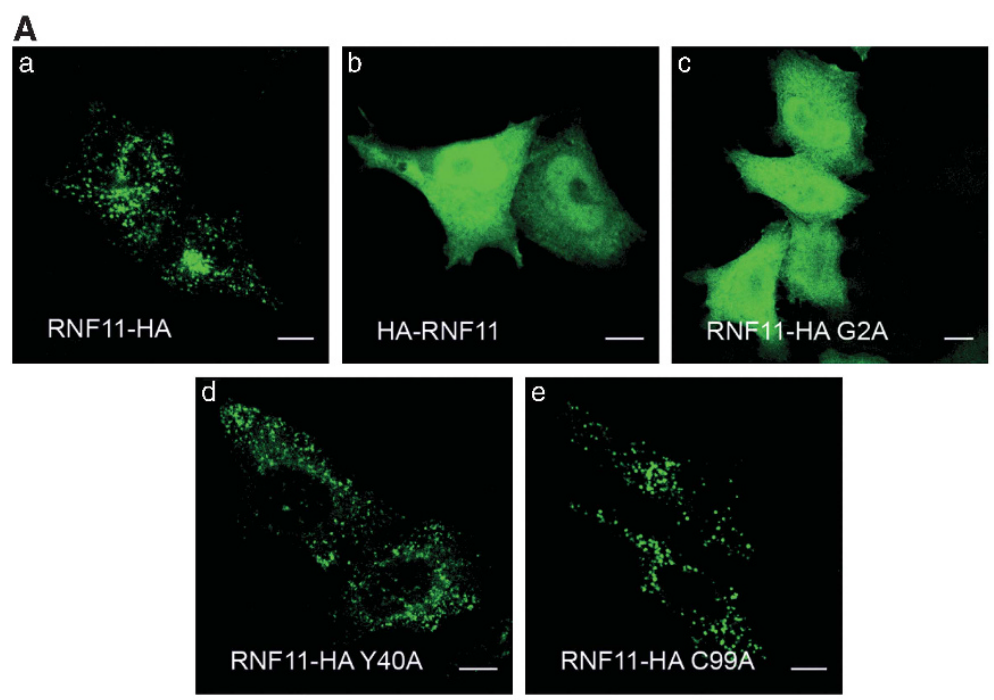

\#1

C

SIRNA

Control
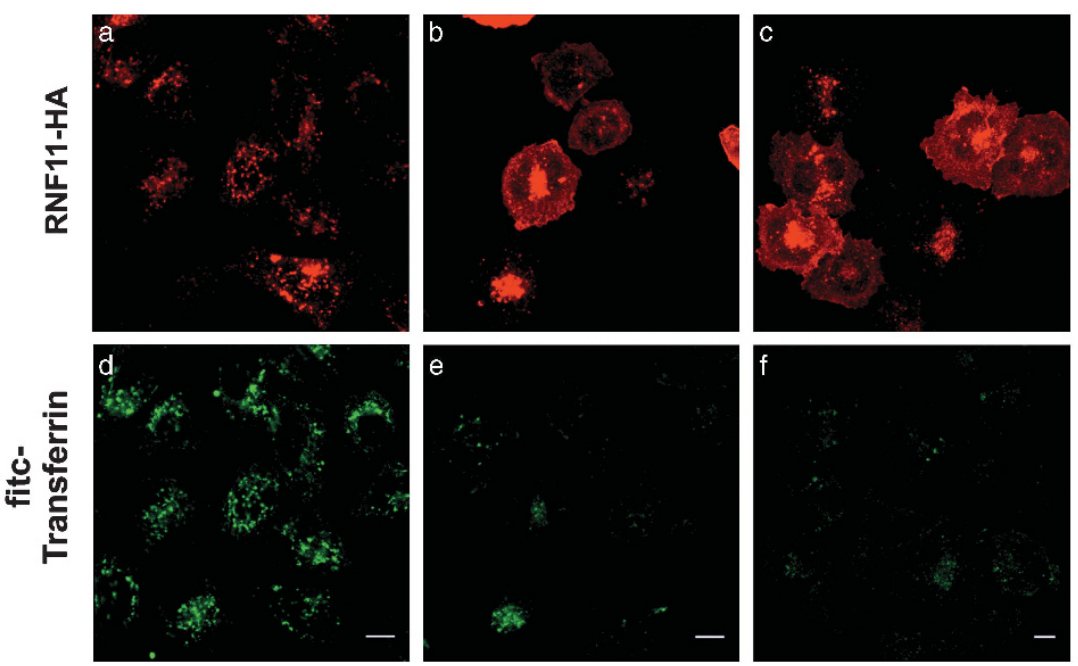

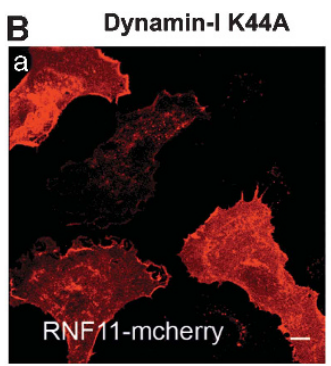

Dynamin-I wt
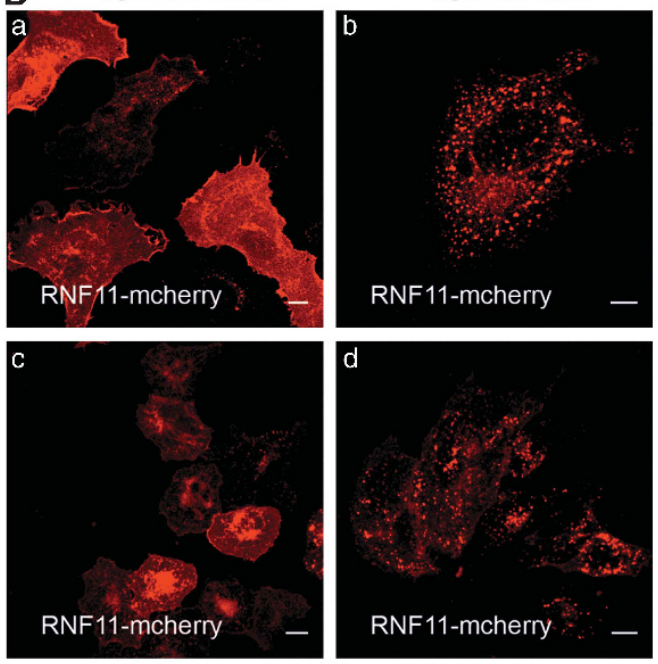

Figure 4. (A) RNF11 is N-terminally myristoylated: HeLa cells were transiently transfected with RNF11-HA (a), HA-RNF11 (b), RNF11-HA G2A (c), RNF11-HA Y40A (d) or RNF11-HA C99A (e). Cells were fixed and processed for indirect immunofluorescence using $\alpha$-HA antibody. (B, C) Inhibition of endocytosis affects RNF11 localization: (A) Human umbilical vein endothelial cells (a, b) or HeLa cells (c, d) were transfected with RNF11-mcherry together with either dominant-negative (K44A) Dynamin-I (a, c) or wild-type Dynamin-I (b, d). (B) Dynamin II-depleted or control cells were transiently transfected with RNF11-HA for $24 \mathrm{~h}$. Fitc-Tf was internalized $72 \mathrm{~h}$ after siRNA transfection, and following fixation cells were processed for indirect immunofluorescence using an $\alpha-\mathrm{HA}$ antibody. At the same time period, cells were lysed and lysates were probed with $\alpha$-Dynamin or $\alpha$-actin antibodies. Scale bars, $10 \mu \mathrm{m}$.

HeLa cells stably expressing approximately endogenous levels of mcherry-TSG101, GFP-SARA was detected in the immunoprecipitates of mcherry-TSG101 (Figure 7b). Likewise, mcherry-Tsg101 was readily detected in the immunoprecipitates of GFP-SARA (Figure 7c). Moreover, ubiquitin-conjugated beads pulled down RNF11-GFP from cell lysates (Figure 7d), and GFP-SARA (Figure 7e), suggesting that RNF11 and SARA might sequester ubiquitinated cargo. Taken together, these results suggest that SARA exhibits all the properties of Hrs concerning ESCRT-0 function, indicating that it could substitute Hrs in some ESCRT-0 complexes.

RNF11 and SARA participate in the sorting functions of ESCRT-0 machinery on epidermal growth factor receptor (EGFR) trafficking One of the sorting cargoes destined for lysosomal degradation by the ESCRT machinery is EGFR (reviewed in Raiborg and Stenmark $\left.^{16}\right)$. Indeed, RNF11 has been shown to influence the levels of
EGFR in cancer cells. ${ }^{20}$ We investigated the effect of RNF11 gene silencing on the EGF-induced degradation of EGFR. At steady state, EGFR levels were higher in the RNF11- and SARA-silenced cells (Figure 8a, left panel and Supplementary Figure 1A). The rate of degradation was significantly slower in the RNF11-silenced compared with the control cells (Figure 8a, right panel). Silencing of SARA exerted an even more pronounced decrease on EGFinduced EGFR degradation compared with RNF11 silencing, as illustrated in the western blot (Figure 8a, left panel and Supplementary Figure 1A) and the degradation curves (Figure 8a, right panel).

Applying the same procedure, we observed that RNF11 or SARA overexpression decreased EGFR degradation in HeLa and LnCAP cells (Figure 8b, $\mathrm{c}$ and Supplementary Figure 1B). In conclusion, silencing and overexpression of RNF11 or SARA decrease EGFinduced EGFR degradation. SARA depletion decreased EGFinduced degradation of EGFR, increasing the intensity and 
a

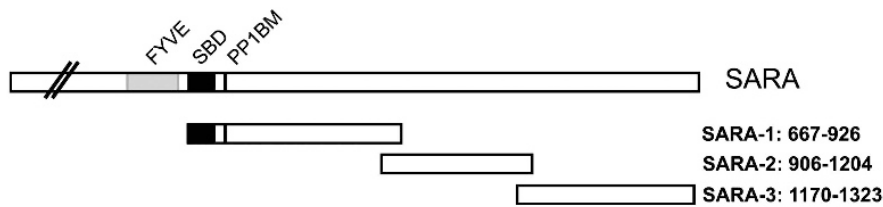

b

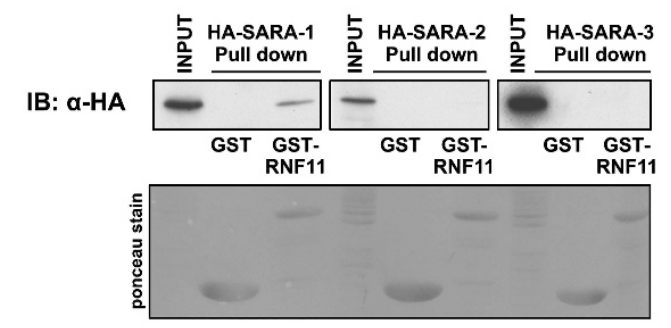

C

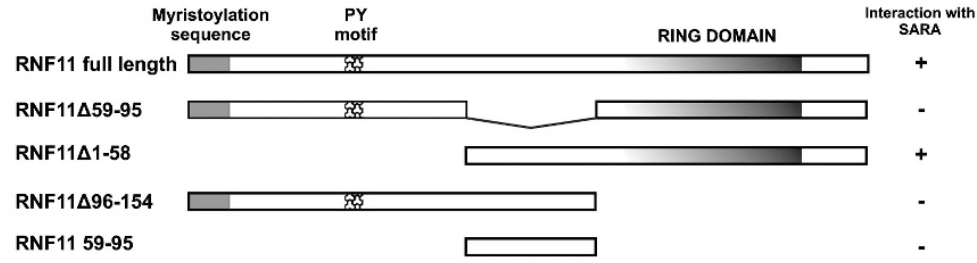

d

\section{e}
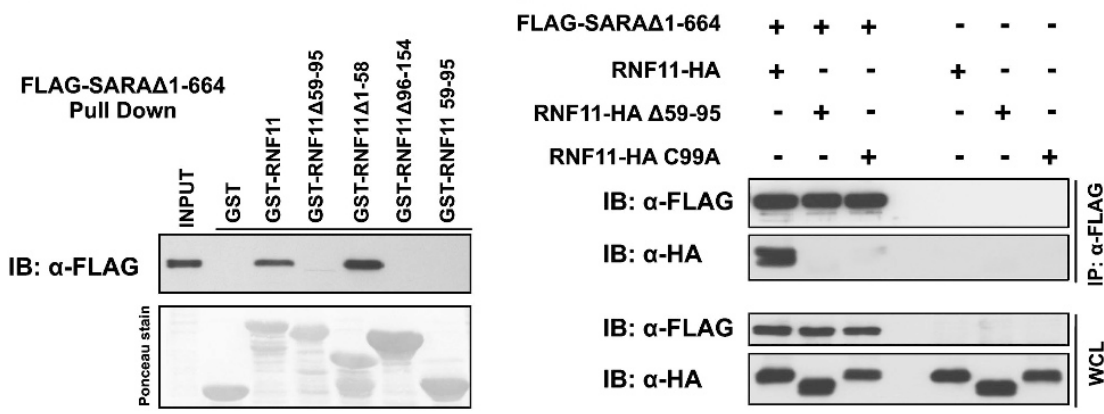

Figure 5. Mapping the RNF11-SARA interaction domains: (a) Domain organization of SARA and constructs used to test RNF11 interaction. (b) HEK293 cell lysates expressing either HA-SARA-1, HA-SARA-2 or HA-SARA-3 were incubated with GST-RNF11 or GST protein alone. The complexes were immunoblotted with an $\alpha-\mathrm{HA}$ antibody. (c) Schematic representation of RNF11 with all known or predicted modular domains or motifs, and constructs generated to test its interaction with SARA. (d) HEK293 cell lysates expressing FLAG-SARA 1 1-664 were incubated in GST protein alone, GST-RNF11 wild-type or GST-RNF11 truncated proteins as outlined in Figure 5c. The complexes were immunoblotted with an $\alpha$-FLAG antibody. (e) HEK293 cells infected with FLAG-SARA $\Delta 1$-664-expressing or control adenoviruses were transfected with the indicated RNF11 constructs. Cell lysates were subjected to immunoprecipitation using $\alpha$-FLAG-affinity conjugated agarose beads. The lower panel in $\mathbf{b}$ and $\mathbf{d}$ shows a ponceau stain of the GST proteins.

duration of ERK1/2 phosphorylation (Figure 8d, lower panel). Also, RNF11 gene silencing appeared to lead to sustained ERK1/2 activation (Figure $8 d$, upper panel), reminiscent to the enhancement of ERK1/2 activation observed during Hrs depletion. ${ }^{21}$ Overexpression of RNF11 and SARA did not lead to visible enhancement of ERK1/2 phosphorylation (Figure 8e).

Enhanced recycling of endocytosed EGF and its receptor $\mathrm{EGFR}^{22}$ which is associated with inhibition of lysosomal receptor trafficking imposed by Hrs or Eps15b depletion, ${ }^{23}$ has been suggested to be responsible for the enhanced ERK1/2 activation. Thus, we investigated the effect of SARA and RNF11 depletion on EGF trafficking. Neither SARA nor RNF11 depletion affected the internalization rate of iodinated EGF (Figure 8f, left panel). ${ }^{24}$ However, although depletion of SARA, RNF11 and Hrs decreased degradation of iodinated EGF (Figure 8f, middle panel), only RNF11 and Hrs depletion led to increased EGF recycling (Figure $8 \mathrm{f}$, right panel). Depletion of SARA did not change the rate of iodinated EGF recycling (Figure 8f, right panel).

To obtain a visual confirmation of the effects of SARA depletion, we investigated the fate of rhodamine-labeled EGF (Rh-EGF) using confocal microscopy after SARA depletion. In the first $10 \mathrm{~min}$, the amount of Rh-EGF internalized in HeLa cells was similar in the presence or depletion of SARA (Supplementary Figure 2A). However, at later time points (30,60 or $120 \mathrm{~min}$ ), the intensity of Rh-EGF fluorescence per cell was clearly higher in SARAdepleted cells (Supplementary Figure 2A). Colocalization of RhEGF with EEA1 revealed that at $30 \mathrm{~min}$, the percentage of Rh-EGF that is localized in early endosomes is clearly increased in SARAdepleted cells (Supplementary Figure $2 \mathrm{~B}$ ). These results suggest that RNF11 depletion delays EGF/EGFR processing in a compartment compatible with recycling that leads to sustained ERK $1 / 2$ activation, whereas SARA depletion seems to 'trap' EGFR in a compartment (positive for EEA1), where recycling cannot occur, but the activity of the EGF/EGFR complex is increased/sustained.

In an attempt to further define the domains of RNF11 involved in degradation of EGFR, we analyzed the effect of RNF11 mutants on the fate of Rh-EGF after 1 and $2 \mathrm{~h}$ following internalization. We compared the effects of GFP alone, RNF11-GFP, RNF11-GFP $\triangle 59-95$, RNF11-GFP C99A and RNF11-GFP Y40A. RNF11-GFP delays the depletion of Rh-EGF as expected, whereas both 
A

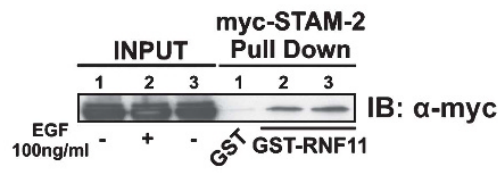

$\stackrel{5}{\underline{5}} \frac{\mathrm{IP}}{\mathrm{HA} \operatorname{IgG}}$

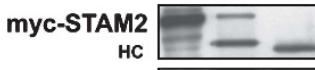

RNF11-HA IB: $\alpha$-myc IB: $\alpha-H A$
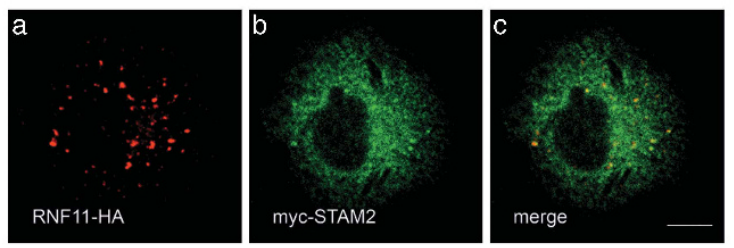

C

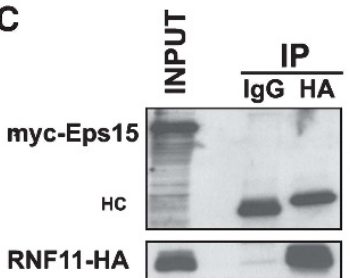
IB: $\alpha-m y c$

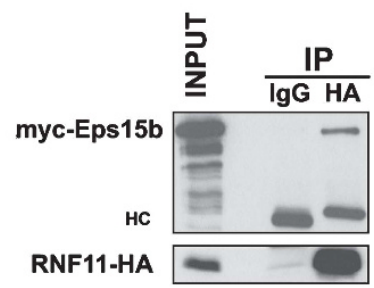
IB: $\alpha-H A$

RNF11-HA

IB: $\alpha$-myc

IB: $\alpha-H A$

B

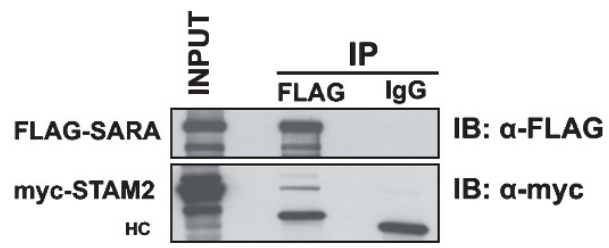

D
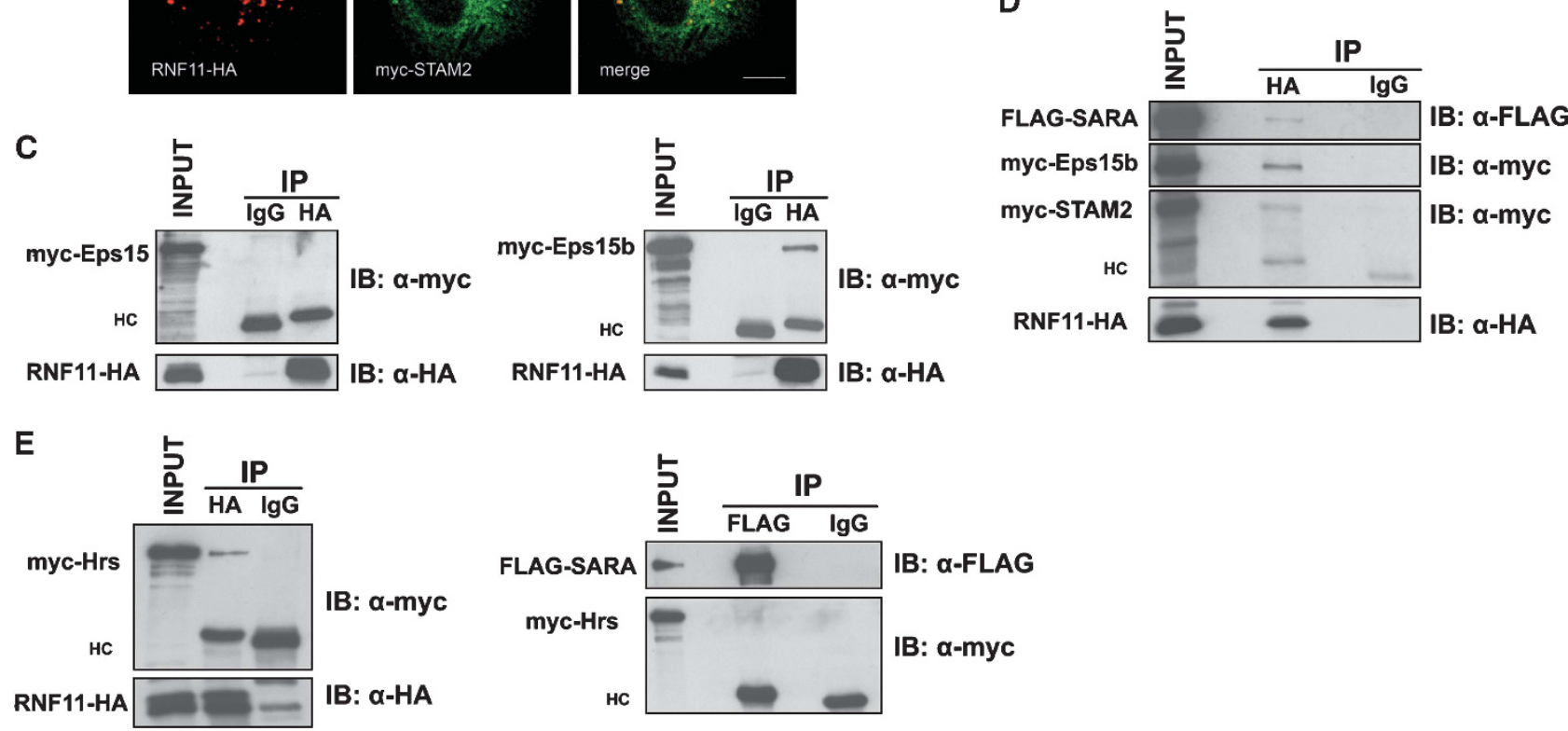

Figure 6. RNF11 and SARA associate with the ESCRT-0: (A) RNF11 and STAM2 interact in vitro and in vivo: (upper left) myc-STAM2 was overexpressed in BHK cells using the T7 RNA polymerase vaccinia virus system, either in the presence or in the absence of EGF (100 ng/ml). Cell lysates were incubated with GST-RNF11 or GST protein alone immobilized on GST beads. The complexes were immunoblotted with an $\alpha$-myc antibody. RNF11-HA and myc-STAM2 (upper right) were overexpressed in BHK cells, and cell lysates were subjected to immunoprecipitation with $\alpha$-HA or rat lgG antibody. The complexes were immunoblotted with an $\alpha$-myc antibody. BHK cells overexpressing RNF11-HA and myc-STAM2 (lower) were fixed and processed for indirect immunofluorescence with $\alpha$-HA and 9 E10 antibodies. Scale bar, $10 \mu \mathrm{m}$. (B) SARA and STAM2 co-immunoprecipitate: FLAG-SARA and myc-STAM2 were overexpressed in BHK cells using the T7 RNA polymerase vaccinia virus system, and cell lysates were subjected to immunoprecipitation with $\alpha$-FLAG or mouse lgG antibodies. The complexes were immunoblotted with an $\alpha$-myc and an $\alpha$-FLAG antibody. (C) RNF11 interacts with Eps15b, but not with Eps15, in vivo: RNF11$\mathrm{HA}$ and myc-Eps15 or myc-Eps15b were overexpressed in BHK cells, and cell lysates were subjected to immunoprecipitation with $\alpha$-HA or rat IgG antibody followed by immunoblot using an $\alpha$-myc and an $\alpha$-HA antibody. (D) SARA, Eps15b, STAM2 and RNF11 are in the same complex: RNF11-HA, myc-Eps15b, myc-STAM2 and FLAG-SARA were overexpressed in BHK cells, and cell lysates were subjected to immunoprecipitation with $\alpha$-HA or rat IgG antibody. After probing with $9 \mathrm{E} 10$ or with $\alpha$-FLAG antibodies, myc-Eps15b, myc-STAM2 and FLAG-SARA were detected only at the $\alpha-\mathrm{HA}$ and not at the lgG control immunoprecipitates. (E) RNF11 interacts but SARA does not interact with Hrs: myc-Hrs and FLAGSARA or RNF11-HA were overexpressed in BHK cells, and cell lysates were subjected to immunoprecipitation with $\alpha$-FLAG or $\alpha$-HA and control IgG antibodies, respectively.

RNF11 $\Delta 59-95$ and RNF11-C99A have a similar effect as the GFP control protein, suggesting that the domains deleted or mutated in these mutants are important for the effect. The PY motif mutant of RNF11 has a similar effect as RNF11 wild-type protein, and we conclude that the PY motif has no regulatory role in this process (Supplementary Figure 4).

\section{DISCUSSION}

We have identified RNF11 as a SARA-interacting protein by means of an $\mathrm{Y} 2 \mathrm{H}$ screen and biochemical validation. RNF11 is a modular 154-aa-containing RING-H2 E3 ligase, harboring a PY motif and a RING-H2 domain. RNF11 interacts with ubiquitin E3 ligases (see below) and numerous other proteins ${ }^{14}$ exerting diverse functions from inhibiting TNF-induced NF- $K B$ signaling ${ }^{25}$ to being proposed as a candidate gene for Parkinson's disease. ${ }^{26}$ RNF11 interacts, via its PY motif, with WW domain-containing proteins, such as the
HECT-type E3 ligases Nedd4, AIP4, Smurf1 and Smurf2, ${ }^{27}$ as well as with Cullin, the core protein in the multi-subunit Skp, Cullin, F-box (SCF) E3 ubiquitin ligase complex. ${ }^{14}$ These E3 ligases ubiquitinate various substrates, but also RNF11 itself. ${ }^{28}$ RNF11 is known to interact also with E2-conjugating enzymes acting as adaptor for activation of E3 ligases. $^{28}$ Interestingly, RNF11 interacts with AMSH, an ubiquitin isopeptidase (a de-ubiquitination enzyme, DUB), recruiting AMSH to Smurf2 for ubiquitination and degradation. ${ }^{15}$ The simultaneous interaction of RNF11 with ubiquitinating and deubiquitinating enzymes indicate that RNF11 may have a role as an ubiquitination fine tuner. These properties rendered RNF11 as an attractive molecule for investigating the significance of its association with SARA in membrane trafficking and signaling.

The interaction of RNF11 with SARA implied that RNF11, or at least a percentage of it, should be localized to the early endocytic compartment. Indeed, we found extensive colocalization of RNF11 
with SARA and early endosomal-specific markers. Moreover, a considerable proportion of RNF11 resides on late endosomes and on the Rab4-positive recycling compartment. These data are in agreement with a very recent report ${ }^{29}$ fully establishing the endocytic localization of RNF11.

The association of RNF11 with SARA was not, however, responsible for the endocytic localization of RNF11. The PY motif and $\mathrm{RING}-\mathrm{H} 2$ domain were excluded also from being responsible for the endosomal localization of RNF11. However, a C-terminal HAtagged RNF11 mutant, in which the critical for acylation glycine at position $2^{30}$ is mutated to alanine (RNF11-HA G2A), did not localize to vesicular structures, instead it exhibited diffuse cytoplasmic and nuclear distribution, confirming recent data on RNF11 myristoylation. $^{29}$ Interestingly, inhibition of endocytosis, significantly, shifted RNF11 localization towards the plasma membrane, suggesting that RNF11, reaching the plasma membrane, undergoes rapid endocytosis and sequestration in the endocytic compartment through unidentified protein/lipid interactions.

In this study, we show that RNF11 interacts with SARA, STAM2 and Eps15b, whereas SARA is associated with RNF11 and STAM2.
In fact, FLAG-SARA, myc-STAM2 and myc-Eps15b were all coimmunoprecipitated by RNF11-HA. This data indicated that RNF11 and SARA might be in complex with ESCRT-0. In confirmation, RNF11 co-immmunoprecipitated Hrs and implied that the wellcharacterized ESCRT-0 complex, comprising Hrs and STAM2, might include also RNF11. However, we were unable to detect any coimmunoprecipitation of SARA with Hrs, a result suggesting that the two FYVE domain proteins, Hrs and SARA, do not exist in the same complex. Taken together, these results indicated that SARA might participate in an alternative complex with RNF11, STAM2 and Eps15b distinct from that formed by Hrs with the same proteins.

In this context, we have additionally shown that SARA interacts with the TSG101 subunit of ESCRT-I, suggesting that it is capable of recruiting ESCRT-I. Indeed, SARA harbors a PTPP sequence compatible with the $\mathrm{P}(\mathrm{S} / \mathrm{T}) \mathrm{XP}$ consensus sequence that binds the ubiquitin E2 variant (UEV) domain of TSG101. ${ }^{31,32}$ Moreover, SARA interacted with clathrin, perhaps, through the LLYMD sequence (aa $637-641) .^{18}$ Thus, SARA exhibits all the key properties of Hrs with regard to ESCRT-0 assembly. These are (i) binding the endosomal a

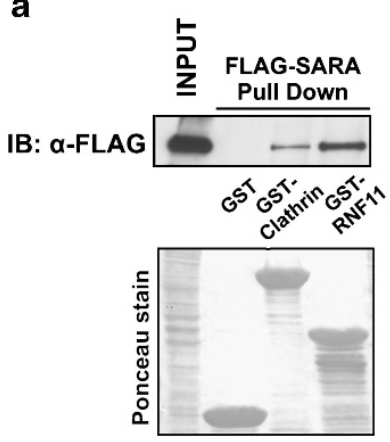

d

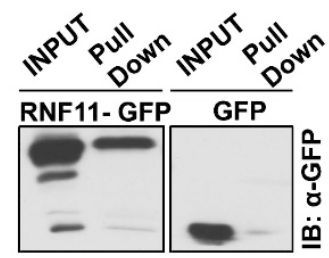

b
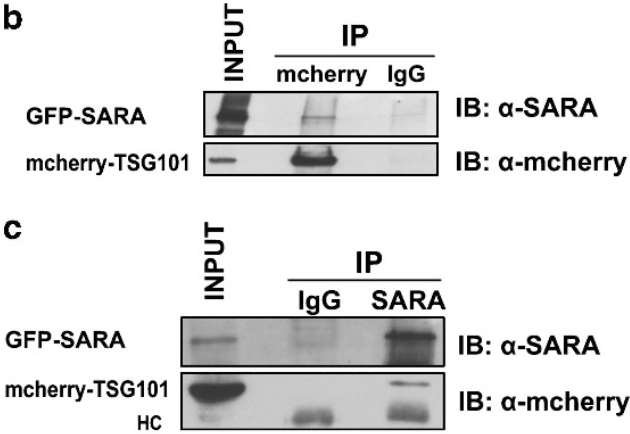

e

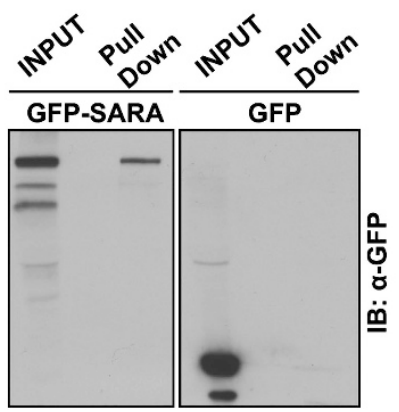

Figure 7. SARA associates with clathrin, TSG101 and ubiquitin: (a) HEK293 cell lysates expressing FLAG-SARA were incubated with GST, GST-clathrin-TD 1-579 or GST-RNF11. The complexes were immunoblotted with an $\alpha$-FLAG antibody. The lower panel shows a ponceau stain of the GST proteins. (b, c) HeLa cells stably expressing mcherry-TSG101 were infected with an adenovirus expressing GFP-SARA. Cells lysates were subjected to immunoprecipitation with an antibody against mcherry and control lgG (b) or against SARA and control lgG (c). (d, e) HEK293 cells were infected with adenoviruses expressing either RNF11-GFP or GFP protein (d) and GFP-SARA or control GFP (e). Cell lysates were incubated with ubiquitin-conjugated agarose beads, and after washes, immunocomplexes were analyzed on an SDS gel and probed with the indicated antibodies.

Figure 8. (a-c) RNF11 and SARA affect EGFR fate: EGFR degradation was monitored (see main text and Materials and methods) in RNF11, control and SARA-depleted $(N=3)(\mathbf{a})$ or in RNF11-GFP- and control-GFP-infected $(N=5)(\mathbf{b})$ or control-GFP- and GFP-SARA-infected HeLa cells $(N=5)$ (c) HeLa cells for the indicated time points. Cells lysates were subjected to SDS electrophoresis and probed with the indicated antibodies. The graphs represent the percentage of remaining EGFR compared with the total (0 min) prior to EGF induction (Error bars are s.e.m. values). In (a) middle panel, the efficiency of RNF11 silencing in HeLa cells measured by quantitative reverse transcription PCR is presented. (d, e) Perturbation of SARA and RNF11 affect EGF signaling: The phosphorylation status of ERK1/2 following treatment with EGF $(100 \mathrm{ng} / \mathrm{ml})$ was monitored using specific $\alpha$-pERK1/2 antibody for the indicated time points in RNF11, control and SARA-depleted (d) or in RNF11-GFP-, GFP-SARA- and control-GFP-infected (e) HeLa cells. (f) RNF11 and SARA differentially affect the recycling of ${ }^{125}$ I-EGF: internalization of ${ }^{125}$ I-EGF $(1 \mathrm{ng} / \mathrm{ml})$ was measured in control, SARA-, RNF11- or HRS-depleted HeLa cells (left). Ratio of internalized to surfacebound EGF was plotted as a function of time. Recycling and degradation of ${ }^{125}$ I-EGF was measured in control, SARA-, RNF11- or HRS-depleted HeLa cells, as described in Materials and methods (middle and right). 
a

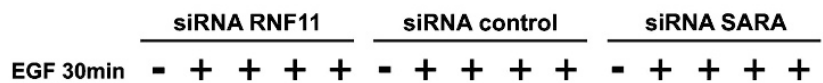
$\begin{array}{llllllllllllllll}\text { Cycloheximide (hours) } & 0 & 0 & 0.5 & 1 & 2 & 0 & 0 & 0.5 & 1 & 2 & 0 & 0 & 0.5 & 1 & 2\end{array}$

IB: $\boldsymbol{\alpha}-E G F R$

IB: $\alpha-S A R A$

IB: $\alpha$-actin
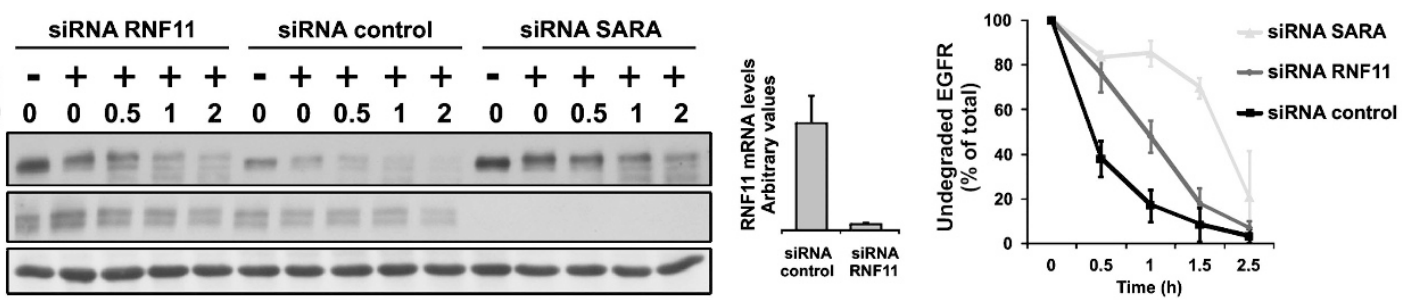

b

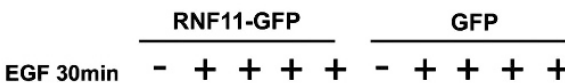

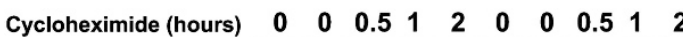

IB: $\alpha$-EGFR $-\infty-\cdots$

IB: $\alpha$-actin $-\infty-\infty-\infty-\infty$

IB: $\boldsymbol{\alpha}$-GFP
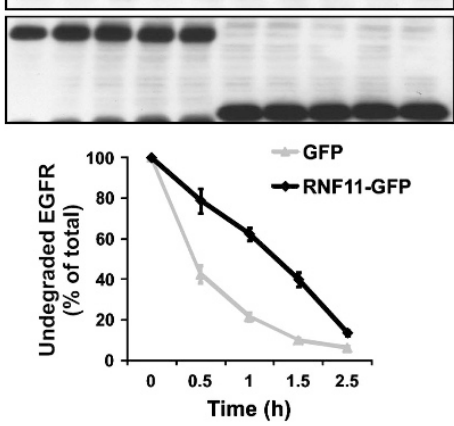

C
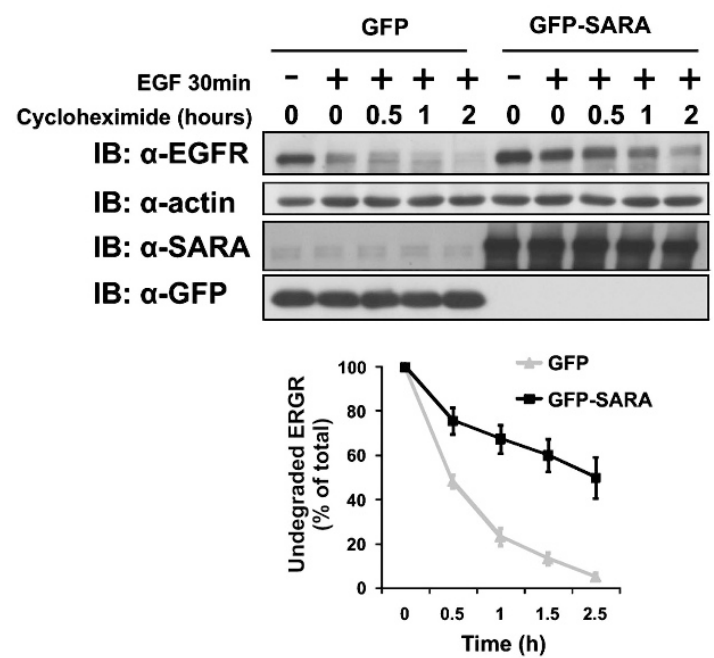

d

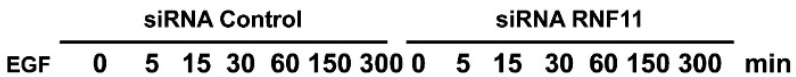

IB: $\alpha-E G F R$

IB: $\alpha-p E R K 1 / 2$

IB: $\boldsymbol{\alpha}$-actin

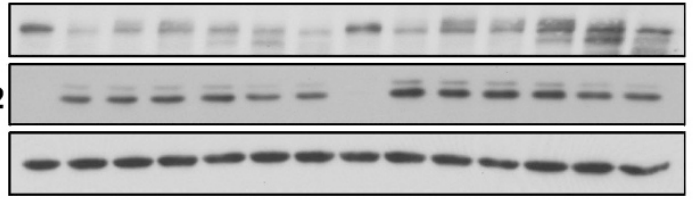

SIRNA Control SIRNA SARA

EGF

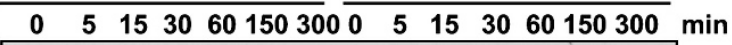

IB: $\alpha-E G F R$

IB: $\alpha-S A R A$

IB: $\alpha-p E R K 1 / 2$

IB: $\alpha$-actin
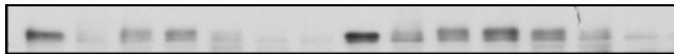

$----\cdots$

$------\infty-1-\infty$

e

\begin{tabular}{llllllllllll}
\cline { 3 - 5 } & 0 & 5 & 15 & 3060150300 & 5 & 5 & 15 & 30 & 60150300 & $\min$
\end{tabular}

IB: $\boldsymbol{\alpha}$-EGFR

IB: $\alpha-p E R K 1 / 2$

IB: $\boldsymbol{\alpha - G F P}$

IB: $\boldsymbol{\alpha}$-actin

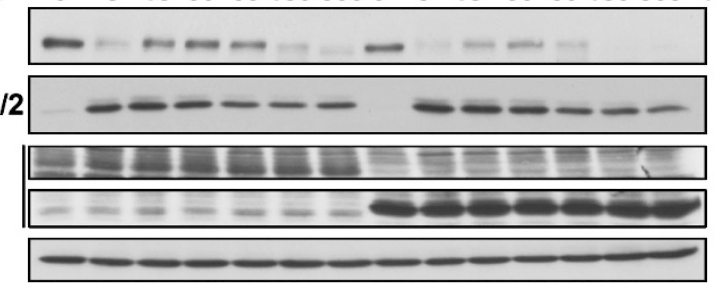

GFP-SARA

IB: $\alpha-E G F R$

IB: $\boldsymbol{\alpha}-S A R A$

IB: $\alpha$-pERK1/2

IB: $\alpha$-actin

IB: $\alpha$-GFP
153060150300 min

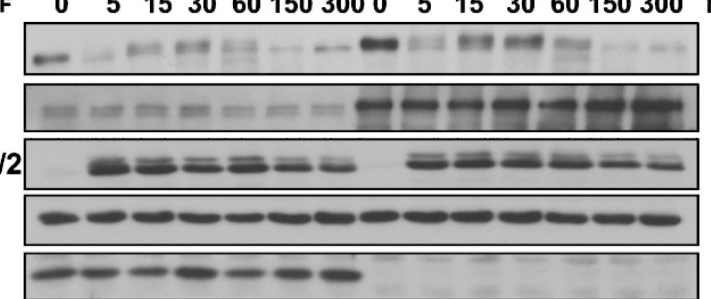

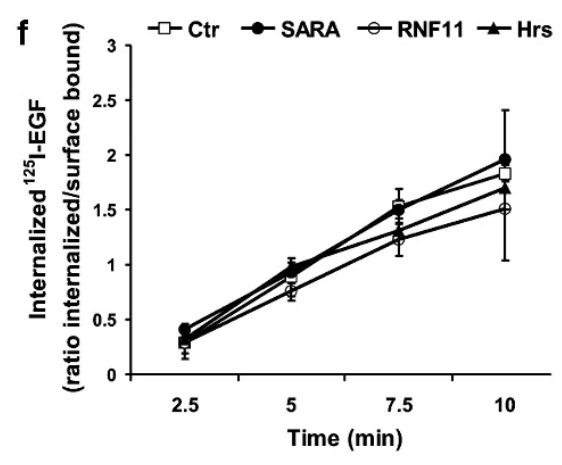

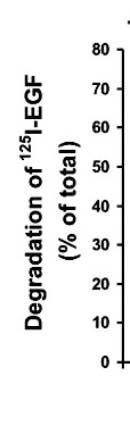

$\square-$ Ctr $\rightarrow$ SARA $\multimap$ RNF11 $\leftarrow$ Hrs
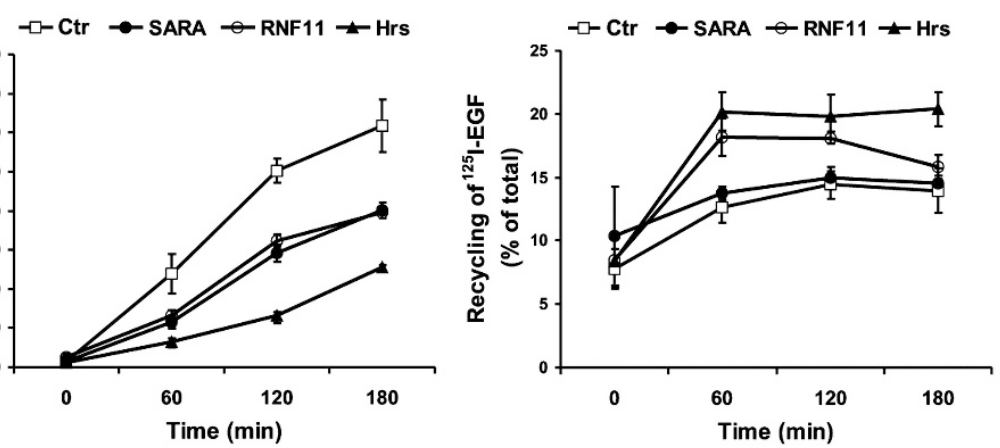
lipid phosphatidylinositol 3-phosphate through its FYVE zinc-finger domain, (ii) binding to STAM2 and Eps15b, (iii) binding the TSG101 subunit to recruit the ESCRT-I complex and (iv) binding to clathrin to increase the concentration of ESCRT-0 in restricted clathrin coats on endosomal membranes. Also, despite the lack of an ubiquitin interacting motif (UIM), GFP-SARA was pulled down by ubiquitin, suggesting that SARA is capable of sequestering ubiquitinated cargo, probably via its associated proteins. It should be noted that several cargos, such as IL-2R $\beta$, are sorted by Hrs from early to late endosomes, independently of UIM-ubiquitin binding. ${ }^{33}$ In these cargos, Hrs recognizes a hydrophobic amino-acid cluster, which is utilized as an ubiquitin-independent sorting signal. ${ }^{34}$ Therefore, SARA exhibits all the structural requirements to form an alternative to Hrs ESCRT-0 complex. Indeed, not only do the Hrs- and TSG101independent pathways exist ${ }^{35,36}$ but also totally ESCRT-independent mechanisms of multivesicular endosome biogenesis have been predicted to occur. ${ }^{37}$

The ESCRT-0 complex consists of Hrs, STAM2 and Eps15b. Interestingly, overexpression or depletion of Hrs and Eps15b, two key components of ESCRT-0, leads to impaired degradation of internalized $\mathrm{EGFR}^{18,23,38,39}$ exhibiting the characteristic bellshaped titration curve of scaffold proteins. ${ }^{40,41}$ Here we show that, similarly to Hrs and Eps15b, depletion and overexpression of RNF11 and SARA impaired EGF-induced EGFR degradation, strongly suggesting that both RNF11 and SARA have an important role in lysosomal sorting of EGFR.

Depletion of Hrs and Eps15b is associated with enhanced recycling of endocytosed EGF and its receptor EGFR, ${ }^{22,23}$ apparently because the ESCRT- 0 complex fails to recognize and cluster $^{42}$ ubiquitinated cargos to prevent their recycling to the plasma membrane. Depletion of components of ESCRT-II and ESCRT-III, when the cargo is already trapped in multivesicular endosomes, does not lead to recycling of the cargo. 22,43 Depletion of RNF11 enhanced EGF recycling, indicating that RNF11 may have a key role in maintaining EGFR ubiquitinated, facilitating cargo recognition and clustering. ${ }^{42}$ Apparently, the RNF11-associated proteins (HECT E3 ligases, SCF E3 ligases and E2-conjugating enzymes), as well as their ability to degrade AMSH (see above), contribute to this activity.

Depletion of SARA does not increase EGF recycling, suggesting an effect at a later stage of the trafficking route. In this respect, depletion of SARA exhibits the same effect as depletion of Vps22/ EAP30 and Vps24/CHMP3, subunits of ESCRT-II and ESCRT-III, respectively. ${ }^{22,43}$ However, there is one important difference. Depletion of SARA enhances ERK1/2 phosphorylation, whereas depletion of Vps22 and Vps24 is without effect on ERK signaling. ${ }^{22,43}$ Because depletion of Hrs (ESCRT-0) and TSG101 (ESCRT-I) enhances ERK $1 / 2$ activation, ${ }^{21,43}$ it seems that termination of EGF signaling occurs before ESCRT-I and -II engagement. Tracing of Rh-EGF in SARA-depleted HeLa cells by confocal microscopy revealed that trafficking of this labeled ligand is delayed in an EEA1-positive early endosomal compartment. Because, ESCRT-I and II localize to the necks of membrane buds, inducing bud formation and confining cargo, ${ }^{42}$ it appears that SARA depletion delays the EGF/EGFR complex in the endosomal compartment just before bud formation, late enough to escape recycling, but still before termination of EGF signaling.

In conclusion, RNF11 interacts with SARA, and both appear to be structurally and functionally associated with the ESCRT-0 complex that regulates lysosomal degradation of EGFRs, a process that has a major role in termination of EGFR signaling. ${ }^{44}$ Thus, perturbation of RNF11 and SARA levels decrease EGFR degradation, thereby generating conditions that may favor their mitogenic signaling. This could be particularly important for mutated EGFR receptors that are constitutively active in certain cancers (reviewed in Mimeault and Batra ${ }^{45}$ ). Indeed, RNF11 is overexpressed in kidney, prostate and invasive breast cancer tumors being particularly high in the latter. ${ }^{27,28}$ Even though the mechanism of RNF11 overexpression in cancer is not clear, high levels of RNF11 in cancer cells may contribute to the process of malignant transformation by decreasing lysosomal degradation of EGFRs, leading to deficient termination of their signaling, as indicated by our study on LnCAP prostate cancer cells. At present, there are no reports regarding the expression status of SARA in cancer cells. Understanding lysosomal degradation of receptors, such as the EGFRs, should allow development of tumor-selective therapeutic approaches targeting EGFR-induced signaling.

\section{MATERIALS AND METHODS}

Cell culture and transfection

Human umbilical vein endothelial cells were isolated, cultured and transfected as previously described. ${ }^{46,47} \mathrm{HeLa}$ and NIH3T3 cells were maintained in Dulbecco's modified Eagle culture medium supplemented with $10 \%$ fetal calf serum and transfected with Effectene (Qiagen, Hilden, Germany). HEK293 were maintained in RPMI1640 culture medium supplemented with $10 \%$ fetal calf serum and transfected with Fugene 6 (Roche, Basel, Switzerland). Culture of BHK cells and overexpression of proteins using the T7 RNA polymerase vaccinia virus system were previous described. ${ }^{48}$ NIH3T3 cells stably lacking SARA were selected and cultured in medium containing $4.5 \mu \mathrm{g} / \mathrm{ml}$ puromycin (Sigma-Aldrich, St Louis, MO, USA) and were transfected with Effectene (Qiagen). HeLa cells stably expressing mcherry-TSG101 were kindly provided by Juan Martin-Serrano, King's College, London, UK, and cultured in medium containing $200 \mathrm{ng} / \mathrm{ml}$ puromycin. A431 cells stably expressing GFP-Rab4 or GFP-Rab11 were kindly provided by Marino Zerial (MPI-CBG, Dresden, Germany), ${ }^{11}$ were maintained in medium supplemented with $0.5 \mathrm{mg} / \mathrm{ml} \mathrm{G} 418$ and transfected with Effectene (Qiagen). The siRNAs ( $20 \mathrm{~nm}$ ) were transfected using RNAimax (Invitrogen, Carlsbad, CA, USA). Conjugated Tf $(50 \mu \mathrm{g} / \mathrm{ml})$ (Molecular Probes, Eugene, OR, USA) was internalized for $30 \mathrm{~min}$ at $37^{\circ} \mathrm{C}$. All media (Invitrogen) were supplemented with $100 \mathrm{U} / \mathrm{ml}$ penicillin, $100 \mu \mathrm{g} /$ $\mathrm{ml}$ streptomycin and $2 \mathrm{~mm}$ glutamine.

\section{Antibodies and recombinant proteins}

hEGF was purchased from ImmunoTools ( $\mathrm{GmbH}$, Friesoythe, Germany) and Tetramethylrhodamine-EGF from Molecular Probes. $\alpha$-EEA1 rabbit polyclonal antibody was kindly provided by Marino Zerial, and an $\alpha$-mcherry mouse monoclonal antibody was kindly provided by Patrick Keller (MPICBG). Mouse $\alpha$-Lamp 1 and $\alpha$-CD63 were purchased from DSHB (lowa City, IA, USA), mouse $\alpha$-EEA 1 and mouse $\alpha$-Dynamin from Transduction Laboratories (Franklin Lakes, NJ, USA), mouse $\alpha$-FLAG M2 from SigmaAldrich, rat $\alpha-\mathrm{HA}$ and mouse $\alpha$-GFP from Roche, rabbit $\alpha$-HA from Babco (Richmond, CA, USA), goat $\alpha$-SARA, rabbit $\alpha$-SARA and goat $\alpha$-EGFR from Santa Cruz Biotechnology (Santa Cruz, CA, USA), mouse $\alpha$-actin from Chemicon (Billerica, MA, USA) and rabbit $\alpha$-pERK1/2 from Cell Signaling (Danvers, MA, USA). A rabbit polyclonal antibody recognizing only overexpressed RNF11 was generated using GST-RNF11 recombinant protein and purified by western strip technique.

Expression plasmids, siRNAs and recombinant adenoviruses The hRNF11 full-length cDNA clone IRATp970A1119D was purchased from imaGenes (Source BioScience UK Ltd., Nottingham, UK). pcDNA3.1 RNF11HA Y40A, pcDNA3.1 RNF11-HA C99A, pcDNA3.1 RNF11, pcDNA3.1 RNF11 HA, pcDNA3 HA-RNF11, pcDNA3.1 RNF11-HA G2A, pcDNA3.1 RNF11-HA $\triangle 59-95$, pEGFP-N1-RNF11, pCDNA3 RNF11-mcherry, pGEM-RNF11-HA, pGEX-6P-1 RNF11, pGEX-6P-1 RNF11 $\Delta 59-95$, pGEX-6P-1 RNF11 $\Delta 1-58$, pGEX-6P-1 RNF11 $\Delta 96-154$ and pGEX-6P-1 RNF11 59-95 were generated by $\mathrm{PCR}$ amplification as outlined in Supplementary Information (Supplementary Table 1). All constructs were sequenced (MWG-Biotech, Ebersberg, Germany). RNF11-Y40A and RNF11-C99A CDNA were provided by Edward Harhaj. pcDNA3 HA-SARA-1, pcDNA3 HA-SARA-2 and pcDNA3 HA-SARA-3 were previously described. ${ }^{49}$ The numbering of SARA is based on Gene Bank sequence AF 104304. pGEM myc-STAM2, pcDNA3 mycEps15 and pcDNA3 myc-Eps15 $\beta$ were as previously described, ${ }^{23,39}$ and pCDNA3 myc-endofin, pEGFP-C2-SARA and pGEM-FLAG-SARA were generated by standard cloning techniques. pCDNA3 myc-Hrs was as described, ${ }^{50}$ whereas GST-clathrin-TD 1-579 was provided by Stuart Kornfeld, Washington University of Medicine, USA. PCB-HA-Dynamin-I K44A, PCB-HA-Dynamin-I and pCDNA3 myc-Rabenosyn- 5 were provided by Savvas Christoforidis, UOI, Greece. pEGFP-C3-Rab11, pEGFP-C3-Rab5a, 
pEGFP-C1-Rab7, pEGFP-N1-Caveolin-1 and pEGFP-C3-Rab4a were from Marino Zerial (MPI-CBG). The siRNAs targeting hRNF11 (ID: 134279, no. 2 134280), hZFYVE9 (ID: 107537, no. 2 107538), hDynamin-2 (ID: 15581, no. 2 15488) and siRNA control (ID: AM4642) were from Applied Biosystems (Carlsbad, CA, USA), whereas the mouse retroviral shRNAmir clone targeting mZFYVE9 (V2MM_205693) was from Thermo Scientific (Waltham, MA, USA). FLAG-tagged SARA $\triangle 1-664$ adenovirus and a control adenovirus expressing GFP alone have been described. ${ }^{3}$ RNF11-GFP, FLAG-SARA and GFP-SARA recombinant adenoviruses were generated and amplified as described (www.coloncancer.org/adeasy.htm), following removal of Pacl site in SARA by site-directed mutagenesis.

Indirect immunofluorescence and statistical analysis

Indirect immunofluorescence, confocal microscopy and image acquisition were carried out as described. ${ }^{3}$ Leica TCS SP5 confocal microscope (Leica Microsystems $\mathrm{GmbH}$, Mannheim, Germany) was used, and objectives HCX PL APO CS $100.0 \times 1.4$ oil and HCX PL APO CS $63.0 \times 1.4$ oil ultraviolet (Leica) were used. Prolong antifade mounting medium was used. The percentage of colocalization was assessed using MotionTrack (Kalaidzidis Y; MPI-CBG) (http://www.kalaimoscope.com/). The number and position $(x-y)$ of the endosomes/vesicles were analyzed and the percentage of colocalization was calculated, with $>50 \%$ overlap of the vesicles considered as colocalized. The total intensity of internalized tetramethylrhodamine-EGF was measured using Image $J(\mathrm{NIH}$, Bethesda, $M D, U S A)$, using the same parameters at all time points.

\section{Pull-down and immunoprecipitations}

GST pull-down assays and immunoprecipitation assays were as described. ${ }^{49}$ Ubiquitin-conjugated agarose bead (Boston Biochem, Cambridge, MA, USA) pull-downs were performed as the GST pull-downs.

\section{EGFR degradation monitoring}

HeLa cells transfected with siRNAs (72h) or infected (24h) with recombinant adenoviruses were serum starved in $0.2 \%$ fetal calf serum for $5 \mathrm{~h}$ and EGF induced $(100 \mathrm{ng} / \mathrm{ml})$ for $30 \mathrm{~min}$. Following induction, cells were chased with cycloheximide (Sigma, St Louis, MO, USA, $25 \mu \mathrm{g} / \mathrm{ml}$ ) in serumstarved conditions for the indicated time points.

\section{Internalization of ${ }^{125} \mathrm{I}$-EGF}

Internalization of $1 \mathrm{ng} / \mathrm{ml}^{125}$-EGF (GE Healthcare, Braunschweig, Germany) was measured upon incubation with EGF as previously described. ${ }^{51}$ Ratio of internalized to surface-bound EGF was plotted as a function of time.

\section{Recycling of ${ }^{125}$ I-EGF}

Recycling and degradation of ${ }^{125}$ I-EGF were performed essentially as described previously. ${ }^{52,53}$ Briefly, cells were incubated with $50 \mathrm{ng} / \mathrm{ml}$ EGF $\left({ }^{125}\right.$ I-EGF: unlabeled EGF, in ratio 1:50) in HEPES containing $5 \%$ fetal calf serum for $15 \mathrm{~min}$ at $37{ }^{\circ} \mathrm{C}$. The cells where washed twice with ice-cold PBS and twice with a glycine-buffered solution $(\mathrm{pH} 3.0)$ to remove surfacebound ${ }^{125}$ I-EGF/EGF. The cells were further incubated in HEPES buffer at $37^{\circ} \mathrm{C}$ for the time periods indicated. Upon the chase, the media were transferred to tubes where proteins were acid precipitated on ice before the samples were centrifuged and separated into supernatant (degraded ${ }^{125}$ I-EGF) and pellet (recycled ${ }^{125}$ I-EGF) fractions. The cells remaining on the plates were lysed with $1 \%$ SDS and represent intracellular ${ }^{125}$ I-EGF. All fractions were counted in a gamma counter, and the distribution between the three fractions was calculated.

\section{CONFLICT OF INTEREST}

The authors declare no conflict of interest.

\section{ACKNOWLEDGEMENTS}

We thank the confocal laser microscope facility of the University of loannina for the use of the Leica TCS-SP scanning confocal microscope. This work was supported by the European Union integrated project ENDOTRACK (EU FP6, LSH-2004-1.1.5-2).

\section{REFERENCES}

1 Conner S, Schmid S. Regulated portals of entry into the cell. Nature 2003; 422 : 37-44.

2 Tsukazaki T, Chiang TA, Davison AF, Attisano L, Wrana J. SARA, a FYVE domain protein that recruits Smad2 to the TGF $\beta$ receptor. Cell 1998; 95: 779-791.

3 Panopoulou E, Gillooly DJ, Wrana JL, Zerial M, Stenmark H, Murphy C et al. Early endosomal regulation of smad-dependent signaling in endothelial cells. J Biol Chem 2002; 277: 18046-18052.

4 Bennett D, Alphey L. PP1 binds sara and negatively regulates dpp signaling in drosophila melanogaster. Nat Genet 2002; 31: 419-423.

5 Hu Y, Chuang JZ, Xu K, McGraw TG, Sung CH. Sara a FYVE domain protein, affects Rab5-mediated endocytosis. J Cell Sci 2002; 115(Pt 24): 4755-4763.

6 Chuang JZ, Zhao Y, Sung CH. SARA-regulated vesicular targeting underlies formation of the light-sensing organelle in mammalian rods. Cell 2007; 130: 535-547.

7 Mu FT, Callaghan JM, Steele-Mortimer O, Stenmark H, Parton RG, Campbell PL et al. EEA1, an early endosome associated protein. EEA1 is a conserved alphahelical peripheral membrane protein flanked cy cysteine 'fingers' and contains a calmodulin-binding IQ motif. J Biol Chem 1995; 270: 13503-13511.

8 Simonsen A, Lippe R, Christoforidis S, Gaullier J-M, Brech A, Callaghan J et al. EEA1 links $\mathrm{PI}(3) \mathrm{K}$ function to rab5 regulation of endosome fusion. Nature 1998; 394: 494-498.

9 Nielsen E, Christoforidis S, Uttenweiler-Joseph S, Miaczynska M, Dewitte F, Wilm $M$ et al. Rabenosyn-5, a novel Rab5 effector, is complexed with hVPS45 and recruited to endosomes through a FYVE finger domain. $J$ Cell Biol 2000; 151: 601-612.

10 Chavrier P, Parton RG, Hauri HP, Simons K, Zerial M. Localization of low molecular weight GTP binding proteins to exocytic and endocytic compartments. Cell 1990; 62: 317-329.

11 Sonnichsen B, De Renzis S, Nielsen E, Rietdorf J, Zerial M. Distinct membrane domains on endosomes in the recycling pathway visualized by multicolor imaging of Rab4, Rab5 and Rab11. J Cell Biol 2000; 149: 901-914.

12 Rothberg KG, Heuser JE, Donzell WC, Ying Y-S, Glenney JR, Anderson RGW. Caveolin a protein component of caveolae membrane coats. Cell 1992; 68: 673-682.

13 Pelkmans L, Kartenbeck J, Helenius A. Caveolar endocytosis of simian virus 40 reveals a new two-step vesicular-transport pathway to the ER. Nat Cell Biol 2001; 5: 473-483.

14 Azmi P, Seth A. RNF11 is a multifunctional modulator of growth factor receptor signalling and transcriptional regulation. Eur J Cancer 2005; 41: 2549-2560.

$15 \mathrm{Li} \mathrm{H}$, Seth A. An RNF11: Smurf2 complex mediates ubiquitination of the AMSH protein. Oncogene 2004; 23: 1801-1808.

16 Raiborg C, Stenmark H. The ESCRT machinery in endosomal sorting of ubiquitylated membrane proteins. Nature 2009; 458: 445-452.

17 Colland F, Jacq X, Trouplin V, Mougin C, Groizeleau C, Hamburger A et al. Functional proteomics mapping of a human signaling pathway. Genome Res 2004; 14: 1324-1332.

18 Raiborg C, Bache KG, Mehlum A, Stang E, Stenmark H. Hrs recruits clathrin to early endosomes. $E M B O$ J 2001; 20: 5008-5021.

19 Bache KG, Brech A, Mehlum A, Stenmark H. Hrs regulates multivesicular body formation via ESCRT recruitment to endosomes. J Cell Biol 2003; 162: 435-442.

20 Chen C, Zhou Z, Liu R, Li Y, Azmi PB, Seth AK. The WW domain containing E3 ubiquitin protein ligase 1 upregulates ErbB2 and EGFR through RING finger protein 11. Oncogene 2008; 27: 6845-6855.

21 Malerod L, Stuffers S, Brech A, Stenmark H. Vps22/EAP30 in ESCRT-II mediates endosomal sorting of growth factor and chemokine receptors destined for lysosomal degradation. Traffic 2007; 8: 1617-1629.

22 Raiborg C, Malerod L, Pedersen NM, Stenmark H. Differential functions of Hrs and ESCRT proteins in endocytic membrane trafficking. Exp Cell Res 2008; 314: 801-813.

23 Roxrud I, Raiborg C, Pedersen NM, Stang E, Stenmark H. An endosomally localized isoform of Eps15 interacts with Hrs to mediate degradation of epidermal growth factor receptor. J Cell Biol 2008; 180: 1205-1218.

24 Collinet C, Stoter M, Bradshaw CR, Samusik N, Rink JC, Kenski D et al. Systems survey of endocytosis by multiparametric image analysis. Nature 2010; 464: 243-249.

25 Shembade N, Parvatiyar K, Harhaj NS, Harhaj EW. The ubiquitin-editing enzyme A20 requires RNF11 to downregulate NF-kappaB signalling. EMBO J 2009; 28: 513-522.

26 Anderson LR, Betarbet R, Gearing M, Gulcher J, Hicks AA, Stefansson $K$ et al. PARK10 candidate RNF11 is expressed by vulnerable neurons and localizes to lewy bodies in parkinson disease brain. J Neuropathol Exp Neurol 2007; 66: 955-964. 
27 Kitching R, Wong MJ, Koehler D, Burger AM, Landberg G, Gish G et al. The RING-H2 protein RNF11 is differentially expressed in breast tumours and interacts with HECT-type E3 ligases. Biochim Biophys Acta 2003; 1639: 104-112.

28 Subramaniam V, Li H, Wong M, Kitching R, Attisano L, Wrana J et al. The RING-H2 protein RNF11 is overexpressed in breast cancer and is a target of smurf2 E3 ligase. Br J Cancer 2003; 89: 1538-1544.

29 Santonico E, Belleudi F, Panni S, Torrisi MR, Cesareni G, Castagnoli L. Multiple modification and protein interaction signals drive the ring finger protein 11 (RNF11) E3 ligase to the endosomal compartment. Oncogene 2010; 29: 5604-5618

30 Resh MD. Fatty acylation of proteins: new insights into membrane targeting of myristoylated and palmitoylated proteins. Biochim Biophys Acta 1999; 1451: 1-16.

31 Bilodeau PS, Winistorfer SC, Kearney WR, Robertson AD, Piper RC. Vps27-Hse1 and ESCRT-I complexes cooperate to increase efficiency of sorting ubiquitinated proteins at the endosome. J Cell Biol 2003; 163: 237-243.

32 Katzmann DJ, Stefan CJ, Babst M, Emr SD. Vps27 recruits ESCRT machinery to endosomes during MVB sorting. J Cell Biol 2003; 162: 413-423.

33 Yamashita Y, Kojima K, Tsukahara T, Agawa H, Yamada K, Amano $\mathrm{Y}$ et al. Ubiquitin-independent binding of Hrs mediates endosomal sorting of the interleukin-2 receptor beta-chain. J Cell Sci 2008; 121(Pt 10): 1727-1738.

34 Amano $Y$, Yamashita $Y$, Kojima K, Yoshino K, Tanaka N, Sugamura K et al. Hrs recognizes a hydrophobic amino acid cluster in cytokine receptors during ubiquitin-independent endosomal sorting. J Biol Chem 2011; 286: 15458-15472.

35 Hislop JN, Marley A, Von Zastrow M. Role of mammalian vacuolar protein-sorting proteins in endocytic trafficking of a non-ubiquitinated $G$ protein-coupled receptor to lysosomes. J Biol Chem 2004; 279: 22522-22531.

36 Gullapalli A, Wolfe BL, Griffin CT, Magnuson T, Trejo J. An essential role for SNX1 in lysosomal sorting of protease-activated receptor-1: evidence for retromer-, Hrs-, and Tsg101-independent functions of sorting nexins. Mol Biol Cell 2006; 17: 1228-1238.

37 Stuffers S, Sem Wegner C, Stenmark H, Brech A. Multivesicular endosome biogenesis in the absence of ESCRTs. Traffic 2009; 10: 925-937.

38 Petiot A, Faure J, Stenmark H, Gruenberg J. PI3P signaling regulates receptor sorting but not transport in the endosomal pathway. J Cell Biol 2003; 162: 971-979.

39 Bache KG, Raiborg C, Mehlum A, Stenmark H. STAM and Hrs are subunits of a multivalent ubiquitin-binding complex on early endosomes. J Biol Chem 2003 278: 12513-12521.

40 Burack WR, Shaw AS. Signal transduction: hanging on a scaffold. Curr Opin Cell Biol 2000; 12: $211-216$
41 Locasale JW, Shaw AS, Chakraborty AK. Scaffold proteins confer diverse regulatory properties to protein kinase cascades. Proc Nat Acad Sci USA 2007; 104: 13307-13312.

42 Wollert T, Hurley $\mathrm{JH}$. Molecular mechanism of multivesicular body biogenesis by ESCRT complexes. Nature 2010; 464: 864-869.

43 Bache KG, Stuffers S, Malerod L, Slagsvold T, Raiborg C, Lechardeur D et al. The ESCRT-III subunit hVps24 is required for degradation but not silencing of the epidermal growth factor receptor. Mol Biol Cell 2006; 17: 2513-2523.

44 Grandal MV, Madshus IH. Epidermal growth factor receptor and cancer: control of oncogenic signalling by endocytosis. J Cell Mol Med 2008; 12: 1527-1534.

45 Mimeault M, Batra SK. Complex oncogenic signaling networks regulate brain tumor-initiating cells and their progenies: pivotal roles of wild-type EGFR, EGFRvIlI mutant and hedgehog cascades and novel multitargeted therapies. Brain Pathol 2011; 21: 479-500.

46 Bagli E, Stefaniotou M, Morbidelli L, Ziche M, Psillas K, Murphy C et al. Luteolin inhibits vascular endothelial growth factor-induced angiogenesis; inhibition of endothelial cell survival and proliferation by targeting phosphatidylinositol 3'-kinase activity. Cancer Res 2004; 64: 7936-7946.

47 Bellou S, Hink MA, Bagli E, Panopoulou E, Bastiaens PI, Murphy C et al. VEGF autoregulates its proliferative and migratory ERK1/2 and p38 cascades by enhancing the expression of DUSP1 and DUSP5 phosphatases in endothelial cells. Am J Physiol Cell Physiol 2009; 297: C1477-C1489.

48 Stenmark H, Bucci C, Zerial M. Expression of Rab GTPases using recombinant vaccinia viruses. Methods Enzymol 1995; 257: 155-164.

49 Sflomos G, Kostaras E, Panopoulou E, Pappas N, Kyrkou A, Politou AS et al. ERBIN is a new SARA-interacting protein: competition between SARA and SMAD2 and SMAD3 for binding to ERBIN. J Cell Sci 2011; 124: 3209-3222.

50 Raiborg C, Bache KG, Mehlum A, Stenmark H. Function of Hrs in endocytic trafficking and signalling. Biochem Soc Trans 2001; 29(Pt 4): 472-475.

51 Johannessen LE, Pedersen NM, Pedersen KW, Madshus IH, Stang E. Activation of the epidermal growth factor (EGF) receptor induces formation of EGF receptorand Grb2-containing clathrin-coated pits. Mol Cell Biol 2006; 26: 389-401.

52 Sorkin AD, Teslenko LV, Nikolsky NN. The endocytosis of epidermal growth factor in A431 cells: a pH of microenvironment and the dynamics of receptor complex dissociation. Exp Cell Res 1988; 175: 192-205.

53 Skarpen $\mathrm{E}$, Johannessen LE, Bjerk K, Fasteng $\mathrm{H}$, Guren TK, Lindeman B et al. Endocytosed epidermal growth factor (EGF) receptors contribute to the EGF-mediated growth arrest in A431 cells by inducing a sustained increase in p21/CIP1. Exp Cell Res 1998; 243: 161-172.

Supplementary Information accompanies the paper on the Oncogene website (http://www.nature.com/onc) 